Les lasers et leurs applications scientifiques et médicales

\title{
Matériaux pour lasers à solide
}

\author{
G. Boulon \\ Laboratoire de Physico-Chimie des Matériaux Luminescents, URA 442 du CNRS, \\ Université Claude Bernard Lyon I, Bât. 205, 43 boulevard du 11 Novembre 1918, \\ 69622 Villeurbanne, France
}

\section{RÉSUMÉ}

Nous nous proprosons de montrer quelle est l'approche des recherches actuelles dans le domaine des lasers à solide qui connaît un net regain d'intérêt à partir de matériaux inorganiques dopés par des ions activateurs et sensibilisateurs comme les ions de transition et les ions de terres rares.

\section{INTRODUCTION}

La recherche de milieux lasers à l'état solide - on dit lasers à solide - connaît un regain d'intérêt certain (1 à 8). Le principal objectif est de trouver de nouveaux matériaux luminescents émettant dans le visible et le proche infrarouge soit sous la forme de raies fines pour les lasers à longueurs d'onde fixes, soit sous la forme de bandes larges pour les lasers à longueurs d'onde accordables. Ces matériaux peuvent en effet recouvrir les bandes spectrables visible et infrarouge utiles pour les applications industrielles, scientifiques, médicales et militaires. A titre d'exemple citons :

- les sources lasers dans les bandes 3-5 $\mu \mathrm{m}$ et 8-12 $\mu \mathrm{m}$ pour les contremesures militaires,

- de 1,3 à $3 \mu \mathrm{m}$ pour les capteurs des systèmes Lidar et les applications médicales,

- l'émission $1,54 \mu \mathrm{m}$ pour les sources lasers à sécurité oculaire nécessitant une radiation inactive sur l'oeil,

- l'émission autour de $1,55 \mu \mathrm{m}$ pour les communications par fibres optiques,

- toutes les sources accordables dans le visible pouvant être doublées par des cristaux non linéaires pour balayer l'uv dont les applications sont nombreuses dans les domaines scientifiques photochimiques et spectroscopiques.

C'est le dopage de matrices cristallines ou vitreuses par des ions activateurs comme les ions de transition et les ions terres rares qui leur confère les propriétés optiques d'absorption et d'émission désirées. Les possibilités de substitution des sites cationiques des réseaux de base sont multiples et dépendent essentiellement des charges et dimensions des cations comparées à celles des ions dopants. Il existe cependant des limitations dans l'application aux cavités lasers associées par exemple à la stabilité thermique du matériau soumis au pompage optique intense des sources excitatrices, ce qui exclut pratiquement les matériaux organiques laissant le champ libre aux matériaux inorganiques.

Ainsi la percée des lasers à solide a été rendue possible par la conjugaison des 
développements des recherches dans les trois domaines de la cristallogénèse, de la spectroscopie des matériaux inorganiques luminescents et des mesures du gain de l'émission stimulée. Le renouveau s'est manifesté en premier lieu par la commercialisation en 1988 et 1989 des lasers accordables dans le proche infrarouge du type saphir dopé titane $\mathrm{AL}_{2} \mathrm{O}_{3}: \mathrm{Ti}^{3+}$ entre 660 et $1060 \mathrm{~nm}$ et du type $\mathrm{MgF}_{2}: \mathrm{Co}^{2+}$ entre 1500 et $2500 \mathrm{~nm}$. Ce renouveau s'est aussi traduit par la mise au point récente de systèmes lasers compacts basés sur des cristaux dopés terres rares pompés par des diodes lasers. Ces diodes lasers apportent en effet une source de pompage remarquable directement sur les premiers niveaux excités de l'ion terre rare activateur $\left(\mathrm{Nd}^{3+}, \mathrm{Tm}^{3+}, \mathrm{Ho}^{3+}, \mathrm{Er}^{3+}, \mathrm{Yb}^{3+}\right)$ réduisant considérablement les pertes d'énergie non radiatives attribuables au pompage lorsqu'il atteint les niveaux excités de plus haute énergie. Malgré cet inconvénient, les niveaux excités supérieurs dans le visible peuvent être aussi pompés dans la plupart des lasers par les lampes à éclairs standard (Xénon par exemple) ainsi que par d'autres lasers dont la commercialisation courante a été un élément moteur du développement de nombreux lasers à solide : ce sont les lasers YAG:Nd doublés à $532 \mathrm{~nm}$ et triplés à $355 \mathrm{~nm}$, les lasers à $\operatorname{Ar}(480$ et $514 \mathrm{~nm})$ à $\mathrm{Kr}(647 \mathrm{~nm})$ ainsi que les lasers à colorants accordables sur l'ensemble du visible. Outre ces aspects liés à l'ingéniérie laser, il convient de mentionner que l'avancement des matériaux lasers doit être aussi reliée à la progression des connaissances dans le domaine de la spectroscopie, surtout dans celui de la dynamique entre les états excités d'un ion (activateur) ou de plusieurs ions (sensibilisateurs et activateurs) lors des processus de transfert d'énergie à la fois radiatifs et non radiatifs.

Dans cet exposé nous rappellerons les grandes dates des matériaux lasers. Nous donnerons ensuite brièvement les principaux intérêts puis nous indiquerons à la fois la nature des ions activateurs et des réseaux hôtes utilisés. Enfin, nous détaillerons quelques résultats et les approches sur les recherches en cours.

\section{LES GRANDES DATES DES MATÉRIAUX LASERS}

Les principales dates des matériaux lasers sont essentiellement focalisées au début des années 60 avec, bien sûr, le premier laser découvert par Maiman en juin 1960 qui est le rubis $\mathrm{AL}_{2} \mathrm{O}_{3}: \mathrm{Cr}^{3+}$ émettant à $694,3 \mathrm{~nm}$ suivi rapidement de nombreux essais avec les ions de transition $\mathrm{Ni}^{2+}, \mathrm{Co}^{2+}, \mathrm{V}^{2+}$ dans les cristaux qui n'ont d'ailleurs pas été exploités de suite à la différence avec l'ion terre rare $\mathrm{Nd}^{3+}$, inséré dans les verres et dans $\mathrm{CaWO}_{4}$ en 1961 et surtout dans le fameux $\mathrm{Y}_{3} \mathrm{Al}_{5} \mathrm{O}_{12}$ (YAG) en 1964 par Guesic, dont l'émission centrée à 1064 nm est, elle, exploitée depuis dans de nombreux lasers scientifiques, industriels et médicaux. Une tentative de lancement vers 1973 de minilasers à base de néodyme comme les composés stoechiométriques $\mathrm{NdP}_{5} \mathrm{O}_{14}$ n'a pas été suivi d'effet en raison de la concurrence duc à la fortc implantation dans l'optoélectronique des lasers à semi-conducteurs qui émettent dans le proche infrarouge. Il a fallu attendre les années 80 pour voir apparaître de nouveaux lasers à solide accordables comme l'alexandrite $\mathrm{BeAl}_{2} \mathrm{O}_{4}: \mathrm{Cr}^{3+}$ malgré les difficultés de synthèse liées à la toxicité du beryllium. Dans ce composé, l'élévation de la température favorise l'émission ${ }^{4} T_{2} \rightarrow{ }^{4} A_{2}$ d'un site $\mathrm{Cr}^{3+}$ à champ faible sous la forme d'une bande large dans le proche infrarouge tandis que le site à champ fort du rubis exclut l'accordabilité et n'autorise que l'émission d'une raie correspondant la transition à ${ }^{2} \mathrm{E} \rightarrow{ }^{4} \mathrm{~A}_{2}$. Les recherches de nouveaux matériaux lasers conduisirent au développement de grenats soit dopés par $\mathrm{Cr}^{3+} \mathrm{Gd}_{3} \mathrm{Sc}_{2} \mathrm{Ga}_{3} \mathrm{O}_{12}: \mathrm{Cr}^{3+}$ ou GSGG: $\mathrm{Cr}^{3+}$ donnant lieu à l'accordabilité de l'émission par la bande ${ }^{4} \mathrm{~T}_{2} \rightarrow{ }^{4} \mathrm{~A}_{2}$ du chrome en substitution sur le site octaédrique en champ faible $\mathrm{Sc}^{3+}$, soit codopés par $\mathrm{Cr}^{3+}-\mathrm{Nd}^{3+}$ ce qui améliore sensiblement le rendement de l'émission laser du néodyme $(7 \%)$ par rapport à celle du YAG:Nd ${ }^{3+}(3,7 \%)$ lorsqu'il est optimisé. Cependant, ce cristal contient du scandium élément rare et cher et, de plus, sa conductibilité thermique n'est pas aussi bonne que celle du YAG. Si bien que depuis 1984 malgré les essais de commercialisation, GSGG: $\mathrm{Cr}^{3+}-\mathrm{Nd}^{3+} \mathrm{n}^{\prime}$ a pas remplacé YAG: $\mathrm{Nd}^{3+}$. D'autres réseaux cristallins ont été dopés par $\mathrm{Nd}^{3+}$ durant cette période. Il serait trop long de tous les mentionner 
et nous ne citerons que les principaux : $\mathrm{LaMgAl}_{11} \mathrm{O}_{19}$ (LMA), $\mathrm{LiYF}_{4}$ (LYF), YAlO 3 (YAP) et $\mathrm{YVO}_{4}$ dont les activations par $\mathrm{Nd}^{3+}$ sont utilisées par des applications particulières.

Outre l'ion $\mathrm{Nd}^{3+}$, les autres terres rares émettant des raies lasers sont $\mathrm{Er}^{3+}$ (autour de 1,55 $\mu \mathrm{m}$ et $2,8 \mu \mathrm{m}$ ), $\mathrm{Tm}^{3+}$ (autour de $1,8 \mu \mathrm{m}$ ), $\mathrm{Ho}^{3+}$ (autour de $2,1 \mu \mathrm{m}$ ) principalement dans $\mathrm{LiYF}_{4}$ et les grenats et plus particulièrement encore dans le YAG tout en bénéficiant de multiples possibilités de transfert d'énergie entre ions sensibilisateurs et activateurs comme ceux entre $\mathrm{Tm}^{3+}-\mathrm{Ho}^{3+}$ ou $\mathrm{Er}^{3+}-\mathrm{Tm}^{3+}-\mathrm{Ho}^{3+}$ ou encore $\mathrm{Cr}^{3+}-\mathrm{Tm}^{3+}-\mathrm{Ho}^{3+}$.

En ce qui concerne les ions de transition, la nouveauté doit être associée avec la commercialisation récente du saphir dopé titane $\mathrm{Al}_{2} \mathrm{O}_{3}: \mathrm{Ti}^{3+}$ accordable dans le proche infrarouge entre 700 et $1100 \mathrm{~nm}$ dans un domaine où les colorants sont photodégradables et n'arrivaient pas à couvrir la plage spectrale. Encore plus récente est l'apparition de lasers accordables entre $1500 \mathrm{~nm}$ et $2500 \mathrm{~nm}$ grâce à la bande d'émission du cobalt dans $\mathrm{MgF}_{2}: \mathrm{Co}^{2+}$.

\section{INTÉRÊTS DES LASERS À SOLIDE}

L'utilisation croissante des lasers à solide est due à plusieurs raisons. D'abord la concentration des ions actifs est plus forte qu'avec les milieux liquides ou gazeux si bien que le volume des monocristaux reste relativement faible sous la forme de barreaux cylindriques et de plaquettes (slabs). Ce sont donc des systèmes compacts. Par ailleurs, ils sont peu fragiles, stables, leur durée de vie n'est théoriquement pas limitée sauf peut-être avec les centres colorés dans les halogénures alcalins. Actuellement, les systèmes compacts sont bien illustrés par YAG:Nd ${ }^{3+}$ pompé par des barrettes de diodes lasers vers $808 \mathrm{~nm}$. En outre on peut extraire des barreaux ou des slabs des puissances moyennes importantes en régime continu : $400 \mathrm{~W}$ avec un barreau YAG: $\mathrm{Nd}^{3+}$ de $16 \mathrm{~cm}$ de longueur et $9 \mathrm{~mm}$ de diamètre et jusqu'à $1800 \mathrm{~W}$ avec un ensemble de 4 barreaux [9]. Les autres raisons sont liées au développement de l'optique non linéaire, aux possibilités de doubler, tripler, additionner plusieurs ondes émettant généralement dans le rouge et le proche infrarouge avec le choix de raies fines et de bandes accordables dans un domaine spectral qui n'a pas pu être pris par les fameux colorants, ce qui conjugue harmonieusement matériaux luminescents lasers et matériaux non linéaires dans des systèmes compacts impossibles à obtenir avec les autres types de lasers conventionnels. Une technologie récente met en œuvre la génération d'ondes par addition et soustraction de fréquences au moyen de matériaux non linéaires. Ces techniques sont, pour la conversion vers les fréquences élevées, la génération du second harmonique, le mélange additif de fréquence et la diffusion Raman stimulée Anti-Stokes tandis que, pour la conversion vers les basses fréquences, ce sont l'oscillation paramétrique optique, le mélange soustractif de fréquences et la diffusion Raman Stimulée Stokes. Enfin, rajoutons que récemment on a vu apparaître des amplificateurs à fibres monomodes (diamètre de l'ordre de $5 \mu \mathrm{m})$ dopées par des traces de terres rares $\left(\mathrm{Nd}^{3+} \mathrm{ou} \mathrm{Er}^{3+}\right.$ ou $\mathrm{Tm}^{3+}$ ) dont les gains autorisent des applications immédiates sur les lignes de communications sous-marines à grande distance afin de concurrencer voire d'éliminer les répéteurs qui sont, on le sait, très onéreux.

\section{LES LASERS À SOLIDE, AVEC QUELS IONS DOPANTS ?}

On rappellera que l'inversion de population entre le niveau émetteur et le niveau final de la transition laser peut être réalisée soit avec un système à 3 niveaux (figure 1a) pour lequel le niveau final est confondu avec le niveau fondamental comme dans $\mathrm{Al}_{2} \mathrm{O}_{3}: \mathrm{Cr}^{3+}\left({ }^{2} \mathrm{E} \rightarrow{ }^{4} \mathrm{~A}_{2}\right.$ à $694,3 \mathrm{~nm})$ ou dans les cristaux et les verres dopés $\mathrm{Er}^{3+}\left({ }^{4} \mathrm{~S}_{3 / 2}{ }^{4} \mathrm{I}_{9 / 2}\right.$ vers $\left.1,66 \mu \mathrm{m}\right)$, soit un système à 4 niveaux (figure $\mathrm{lb}$ ) beaucoup plus aisé à mettre en oeuvre en raison d'un seuil beaucoup plus faible que celui à 3 niveaux. C'est en fait le modèle de tous les autres centres activateurs : citons les niveaux électroniques des ions terres rares dont le meilleur exemple est $\mathrm{Nd}^{3+}$ pour lequel la différence entre (2) et (1) est voisine de $2000 \mathrm{~cm}^{-1}$ (environ $10 \mathrm{kT}$ ) laissant la population de (2) pratiquement nulle avant pompage. C'est également le modèle pour les 


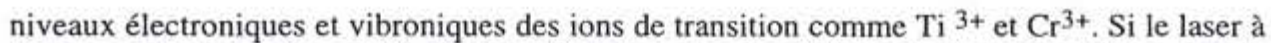
rubis a tendance à être retiré du marché c'est parce que justement c'est un système à trois niveaux peu efficace sous pompage par les lampes émettant dans le visible ce qui conduit à des pertes non radiatives importantes entre les niveaux excités avant l'émission du niveau ${ }^{2} \mathrm{E}$ laquelle par ailleurs a tendance à repeupler directement le niveau fondamental ${ }^{4} \mathrm{~A}_{2}$.

Cependant ce modèle à 3 niveaux peut être efficace quand on a la possibilité de pomper près du niveau émetteur avec un laser pompe ou une diode laser comme c'est le cas avec $\mathrm{Er}^{3+}$ pompé à $1485 \mathrm{~nm}$ et émettant à $1535 \mathrm{~nm}$ vers le niveau fondamental.

Parmi tous les ions luminescents les recherches portent essentiellement sur ceux qui peuvent être pompés efficacement dans le visible et le proche infrarouge donnant lieu à des émissions présentant des rendements de fluorescence élevés dans les fenêtres de transparence des cristaux pour qu'elles ne soient pas réabsorbées par le réseau cristallin lui-même. A ce propos, il sera difficile d'utiliser l'ultraviolet qui engendre des centres colorés et provoque des dommages aux cristaux comme la solarisation. On comprend alors tout l'intérêt qu'il y a de bien connaître la spectroscopie d'absorption et d'émission aussi bien du réseau hôte que de l'ion activateur. Rajoutons que la connaissance de la spectroscopie des ions luminescents permet d'envisager des transferts d'énergie entre un ou plusieurs ions afin d'une part d'améliorer les rendements de l'intensité lumineuse et d'autre part d'ajuster les sources de pompage commerciales aux niveaux d'absorption des états excités.

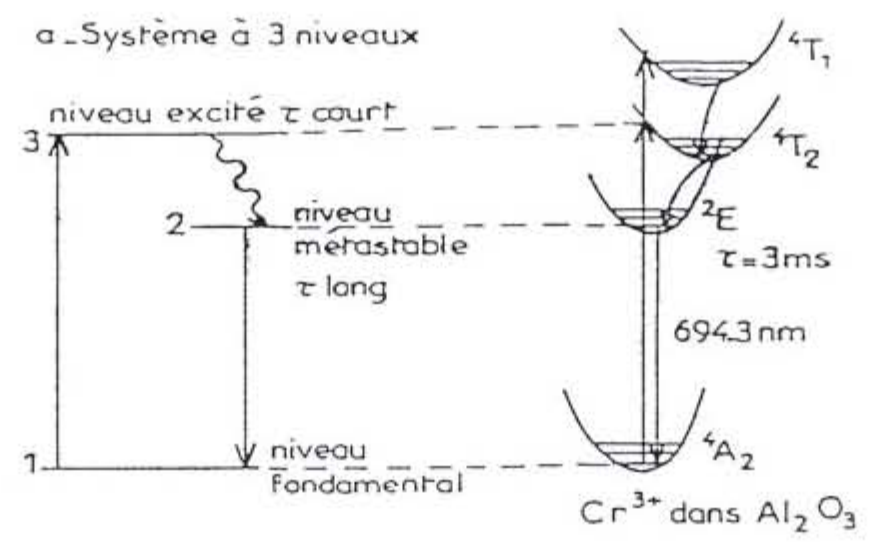

b-Système à 4 niveoux

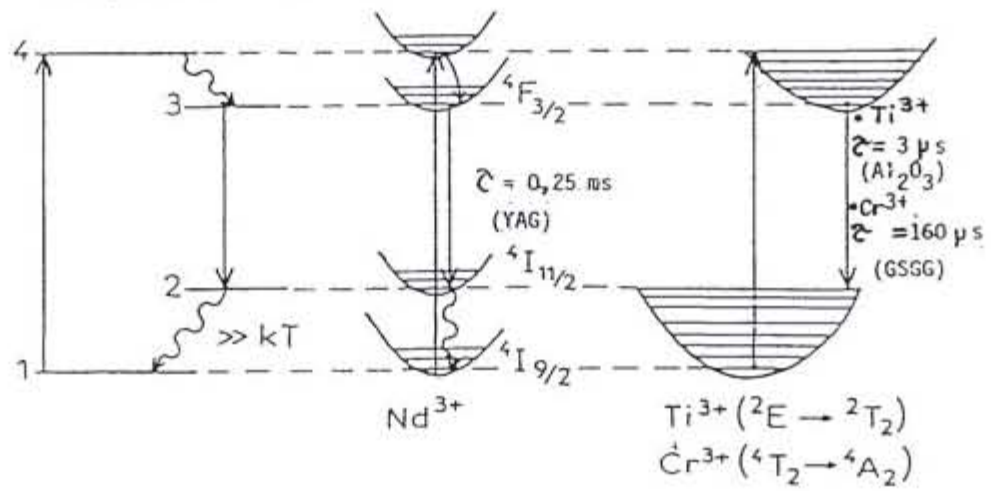

Figure 1 : Diagramme des niveaux d'énergie et couches de configuration (énergie potentielle du centre activateur entouré de ses plus proches voisins) des systèmes à 3 et 4 niveaux susceptibles de donner l'effet laser. 
Actuellement ces principaux dopants sont :

-les ions de transitions: $\mathrm{Ti}^{3+}\left(3 \mathrm{~d}^{1}\right), \mathrm{Cr}^{4+}\left(3 \mathrm{~d}^{2}\right) \mathrm{Cr}^{3+}$ et $\mathrm{V}^{2+}\left(3 \mathrm{~d}^{3}\right), \mathrm{Co}^{2+}\left(3 \mathrm{~d}^{7}\right)$ et $\mathrm{Ni}^{2+}\left(3 \mathrm{~d}^{8}\right)$.

-les ions terres rares $\mathrm{Pr}^{3+}\left(4 \mathrm{f}^{2}\right), \mathrm{Nd}^{3+}\left(4 \mathrm{f}^{3}\right), \mathrm{Dy}^{3+}\left(4 \mathrm{f}^{9}\right), \mathrm{Ho}^{3+}\left(4 \mathrm{f}^{10}\right), \mathrm{Er}^{3+}\left(4 \mathrm{f}^{11}\right)$, $\mathrm{Tm}^{3+}\left(4 \mathrm{f}^{12}\right)$.

\section{LES LASERS À SOLIDE, AVEC QUELS TYPES DE MATÉRIAUX INORGANIQUES ?}

Là aussi les possibilités sont nombreuses, limitées certes par la substitution entre cations du réseau et activateurs qui doivent avoir de préférence même charge et même rayon d'ion, ainsi que par la cristallogénèse du monocristal laser. De plus, ces matériaux ne doivent pas seulement remplir les conditions imposées par les propriétés optiques adaptées aux lasers à savoir large fenêtre de transparence entre l'absorption fondamentale dans l'uv et l'absorption des groupements moléculaires dans l'infrarouge afin que le dopage apporte des bandes d'absorption et d'émission bien localisées dans le visible et le proche infrarouge. Actuellement on connaît des émissions de $200 \mathrm{~nm}$ à $5150 \mathrm{~nm}(5,15 \mu \mathrm{m})$. Ils doivent, en effet, également présenter d'excellentes qualités mécaniques et thermiques en vue de leur pompage par des flux intenses. Il n'est donc pas étonnant de retrouver les cristaux de joaillerie sous leur forme synthétique comme le rubis $\mathrm{Al}_{2} \mathrm{O}_{3}: \mathrm{Cr}^{3+}$, l'émeraude $\mathrm{Be}_{3} \mathrm{Al}_{2}\left(\mathrm{SiO}_{3}\right)_{6}: \mathrm{Cr}^{3+}$, l'alexandrite $\mathrm{BeAl}_{2} \mathrm{O}_{4}: \mathrm{Cr}^{3+}$, les spinelles dopés $\mathrm{Cr}^{3+}$, les grenats dopés $\mathrm{Cr}^{3+}, \mathrm{Nd}^{3+}$ comme le fameux $\mathrm{Y}_{3} \mathrm{Al}_{5} \mathrm{O}_{12}$ et le saphir dopé $\mathrm{Ti}^{3+}$.

En fait, on rencontre tous les types de structures cristallographiques pourvu qu'elles contiennent soit des cations trivalents (comme $\mathrm{Al}^{3+}$ ) ou divalents (comme $\mathrm{Mg}^{2+}$ ) susceptibles d'être subtitués par les ions de transition divalents ou trivalents, soit dans le cas de la substitution par des ions terres rares trivalents, $\mathrm{Sc}^{3+}, \mathrm{Y}^{3+}, \mathrm{La}^{3+}$ et évidemment les 14 autres éléments de la famille des lanthanides. Les principales structures sont le corindon $\mathrm{Al}_{2} \mathrm{O}_{3}$, l'olivine avec $\mathrm{BeAl}_{2} \mathrm{O}_{4}$ ou la forstérite $\mathrm{Mg}_{2} \mathrm{SiO}_{4}$, la péroskite $\mathrm{YAlO}_{3}$ (YAP), la scheelite comme $\mathrm{CaWO}_{4}$ ou $\mathrm{LiYF}_{4}$ (YLF), la magnétoplombite $\mathrm{LaMgAl}_{11} \mathrm{O}_{19}$ (LMA), l'eulytine $\mathrm{Bi}_{4} \mathrm{Ge}_{3} \mathrm{O}_{12}$ (BGO), le rutile $\mathrm{MgF}_{2}$, l'ultraphosphate $\mathrm{NdP}_{5} \mathrm{O}_{14}$ et les grenats $\mathrm{A}_{3} \mathrm{~B}_{2} \mathrm{C}_{3} \mathrm{O}_{12}$ comme $\mathrm{Y}_{3} \mathrm{Al}_{5} \mathrm{O}_{12}$ (YAG), $\mathrm{Gd}_{3} \mathrm{Sc}_{2} \mathrm{Ga}_{3} \mathrm{O}_{12}$ (GSGG), $\mathrm{Gd}_{3} \mathrm{Ga}_{5} \mathrm{O}_{12}$ (GGG) ou encore (Gd, $\left.\mathrm{Ca}\right)_{3}(\mathrm{Ga}, \mathrm{Mg}, \mathrm{Zr})_{5} \mathrm{O}_{12}$ appelés grenats subsitués et des germanates du type $\mathrm{Ca}_{3} \mathrm{Ga}_{2} \mathrm{Ge}_{3} \mathrm{O}_{12}, \mathrm{CaY}_{2} \mathrm{Mg}_{2} \mathrm{Ge}_{3} \mathrm{O}_{12}$ (CAMGAR). On trouvera dans les ouvrages de M.J Weber et A. Kaminskii des listes exhaustives des réseaux hôtes [1-2].

Une voie de recherche actuelle consiste à doper, par les ions fluorescents habituels, les solutions solides les plus connues pouvant être obtenues sous la forme de monocristaux à l'échelle centimétrique par des fusions congruentes, à des températures inférieures à environ $1850^{\circ} \mathrm{C}$, autorisant l'utilisation de creuset d'irridium. Au-delà on peut espérer développer les méthodes de croissance cristalline de zone flottante par fusion au moyen de lasers continus à $\mathrm{CO}_{2}$ si on se restreint à des fibres cristallines assez faciles à synthétiser et utiles pour l'obtention et l'étude spectroscopique de petits cristaux présentant des points de fusion supérieurs à $1900^{\circ} \mathrm{C}$.

A titre d'exemple les recherches de solutions solides sont actives avec le dopage par le chrome $\mathrm{Cr}^{3+}$ des sites octaédriques du spinelle $\mathrm{Mg}_{2} \mathrm{Al}_{2} \mathrm{O}_{4}$, des cordiérites de magnésium sans fer, des olivines comme la forstérite $\mathrm{Mg}_{2} \mathrm{SiO}_{4}$ qui semble conduire à la présence de chrome $\mathrm{Cr}^{4+}$ en plus de $\mathrm{Cr}^{3+}$ donnant un laser accordable qui s'étend jusqu'à $1,2 \mu \mathrm{m}$ (figure 2a) et qui relance la cristallogénèse et les propriétés optiques des cristaux dopés par $\mathrm{Cr}^{3+}$ comme par exemple dans les grenats qui peuvent être codopés par les couples $\mathrm{Cr}^{4+}-\mathrm{Mg}^{2+}$ ou $\mathrm{Cr}^{4+} \mathrm{Ca}^{2+}$ (voir figure $2 \mathrm{~b}$ ). Il serait probablement très intéressant d'explorer parmi les solutions solides les plus importantes dans les minéraux, toutes celles susceptibles de contenir $\mathrm{Al}^{3+}$ en position octaédrique pour la substitution par $\mathrm{C}^{3+}$ ainsi que $\mathrm{Si}^{4+}$ pour la substitution prometteuse par $\mathrm{Cr}^{4+}$ dont on confirme la spectroscopie de ce cation dans les minéraux de façon à étendre notablement le domaine d'émission du cristal laser. Des recherches portent ainsi sur les silicates 
comme $\mathrm{Y}_{2} \mathrm{SiO}_{5}$ dont le site $\mathrm{Y}^{3+}$ peut être substitué avec plusieurs activateurs terres rares [10].

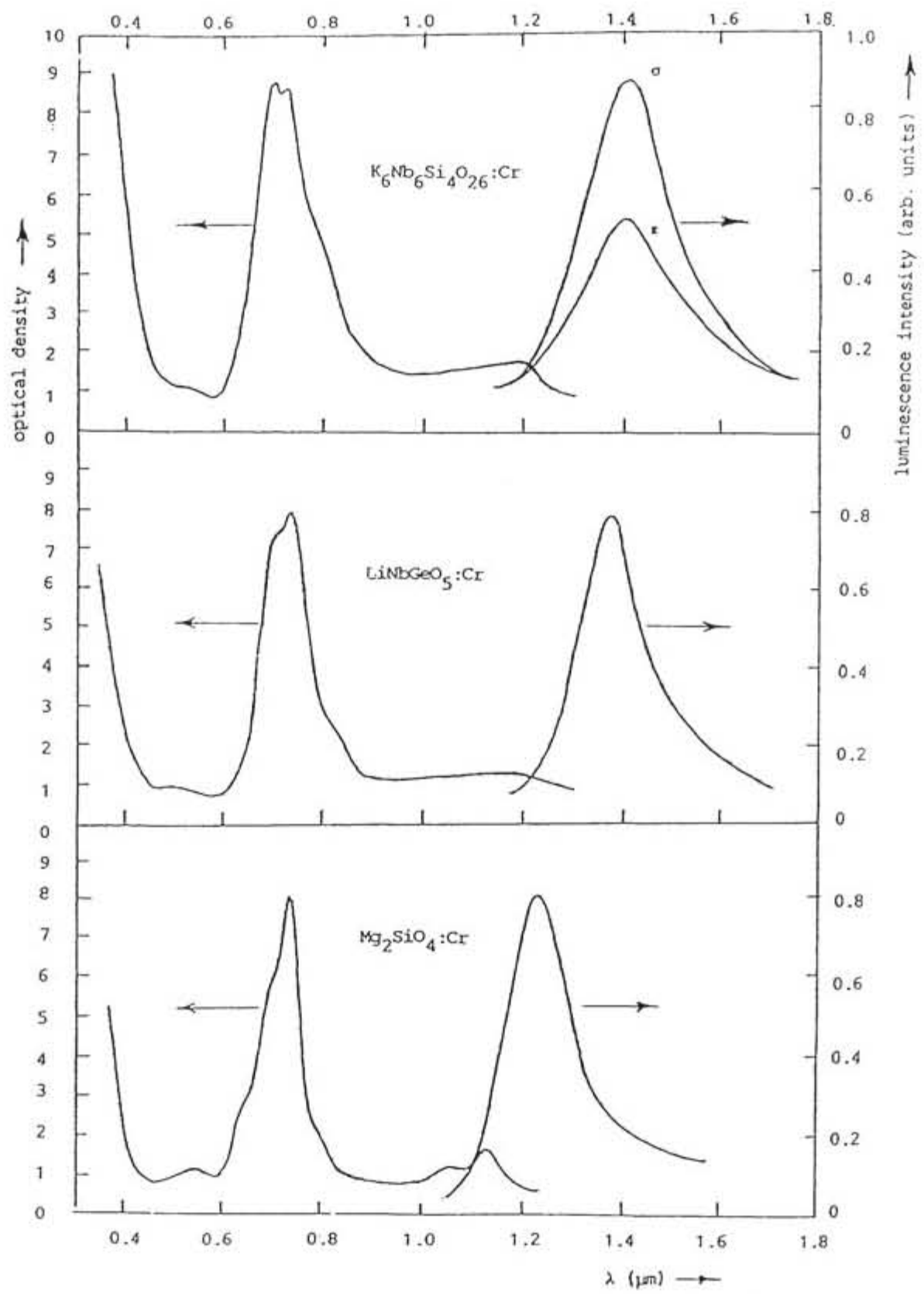

Figure 2a : Spectres d'absorption et d'émission de quelques cristaux dopés par l'ion $\mathrm{Cr}^{4+}$ à la température ambiante [24] 


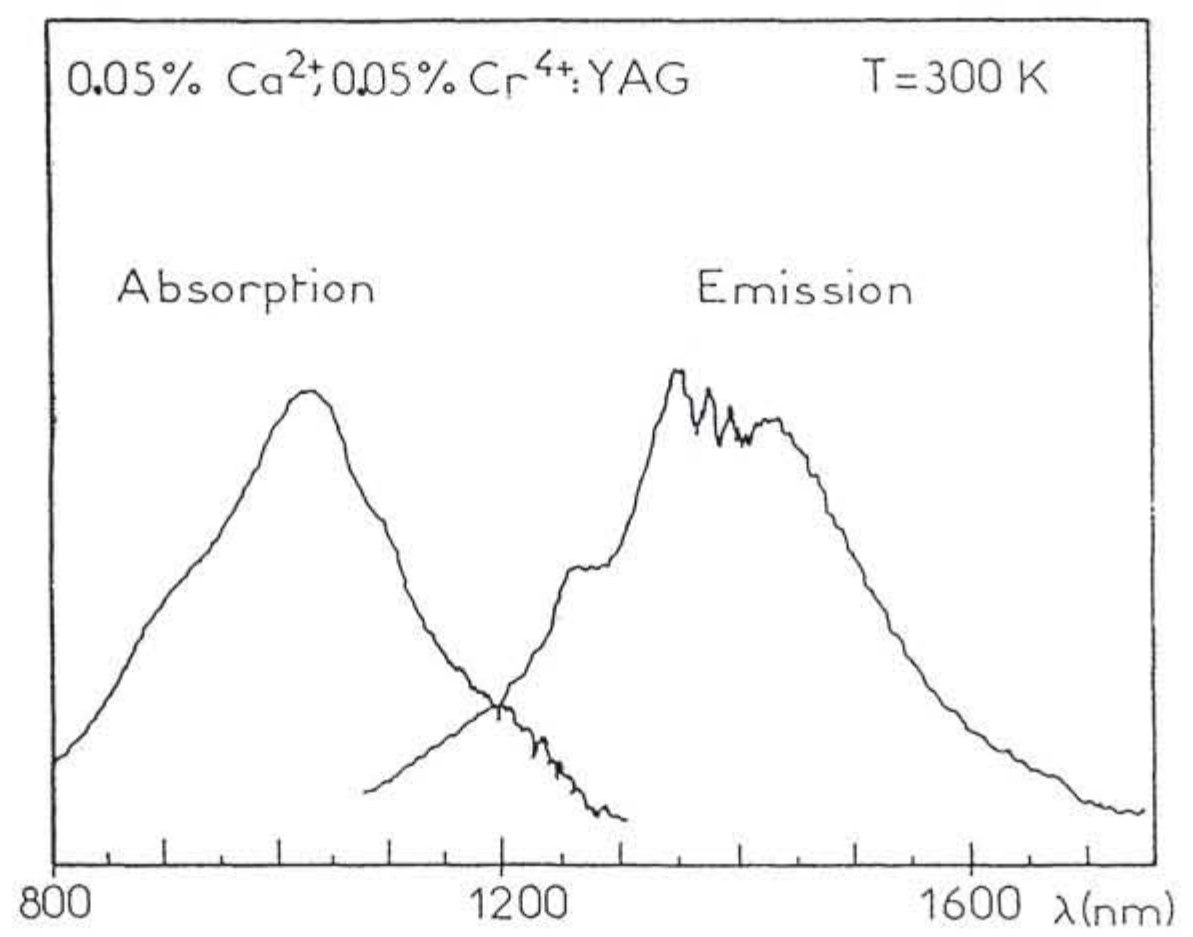

Figure 2b : Spectres d'absorption et d'émission de l'ion $\mathrm{Cr}^{4+}$ dans $\mathrm{Y}_{3} \mathrm{Al}_{5} \mathrm{O}_{12}$ à la température ambiante.

\section{LES IONS DE TRANSITION COMME IONS ACTIFS}

Les matériaux lasers contenant des ions de transition sont des monocristaux d'oxydes ou de fluorures. Ils constituent essentiellement la famille des lasers à longueurs d'onde accordables sur les bandes d'émission vibroniques de ces ions. Les électrons de la configuration $3 \mathrm{~d}$ qui sont impliqués dans les transitions optiques sont fortement couplés aux vibrations du réseau et les spectres d'émission apparaissent sous la forme de bandes larges utiles pour l'accordabilité de l'effet laser. Nous avons rassemblé sur le tableau 1 les principales caractéristiques de ces types de lasers en se limitant à la température ambiante qui reste évidemment l'un des paramètres de sélection pour le développement des matériaux pour l'optique. Ceci limite considérablement leur nombre à cause de l'effet d'extinction thermique de la fluorescence liée à l'interaction électronphonon et élimine une grande quantité qui émettent l'effet laser aux basses températures.

\subsection{L'ion $\mathrm{Cr}^{3+}\left(3 \mathrm{~d}^{3}\right)$}

Cet ion peut donner lieu à deux types de transitions interconfigurationnelles $\left(e_{g}\right.$ et $t_{2 g}$ ) et intraconfigurationnelles à $\mathrm{t} 2 \mathrm{~g}$, celles-ci constituant la seule exception à la règle du fort couplage électron-phonon. Elle est de taille puisqu'elle est constituée par la première mise en évidence, en 1960 , curieusement du système laser à 3 niveaux et non pas à 4 niveaux avec la raie ${ }^{2} \mathrm{E} \rightarrow{ }^{4} \mathrm{~A}_{2}$ dont la présence s'explique sur la figure 3 par la position de l'ion $\mathrm{Cr}^{3+}$ dans un site octaédrique 
à champ fort, le niveau ${ }^{2} \mathrm{E}$ étant alors le plus bas des niveaux excités qui émet après pompage sur les bandes larges ${ }^{4} \mathrm{~T}_{2},{ }^{4} \mathrm{~T}_{1}$ et relaxations non-radiatives vers ${ }^{2} \mathrm{E}$. Par contre, si la distance entre $\mathrm{Cr}^{3+}$ et les $\mathrm{O}^{2-}$ les plus proches voisins de l'octaèdre de coordination augmente avec l'accroissement du paramètre de maille du réseau, l'intensité du champ cristallin diminue abaissant le niveau ${ }^{4} \mathrm{~T}_{2}$ au voisinage voire sous le niveau ${ }^{2} \mathrm{E}$ ce qui donne lieu à la bande large d'émission ${ }^{4} \mathrm{~T}_{2} \rightarrow{ }^{4} \mathrm{~A}_{2}$ qui s'interprète alors dans le système à 4 niveaux. C'est ce dernier cas qui correspond le mieux à la série des grenats de structure cubique dont le paramètre de maille $\mathrm{a}_{\mathrm{o}}$ varie de la manière suivante : $12,01 \AA$ dans le YAG, $12,38 \AA$ dans $\mathrm{Gd}_{3} \mathrm{Ga}_{5} \mathrm{O}_{12}, 12,47 \AA$ dans GGG ( $\mathrm{Ca}, \mathrm{Zr}$ ) et $12,55 \AA$ dans GSGG [11]. Il en résulte une évolution progressive du spectre d'émission à la température ambiante allant du mélange de la raie ${ }^{2} \mathrm{E} \rightarrow{ }^{4} \mathrm{~A}_{2}$ et de la bande ${ }^{4} \mathrm{~T} 2 \rightarrow{ }^{4} \mathrm{~A}_{2}$ lorsque les niveaux ${ }^{2} \mathrm{E}$ et ${ }^{4} \mathrm{~T}_{2}$ sont proches, ${ }^{2} \mathrm{E}$ restant en dessous de ${ }^{4} \mathrm{~T}_{2}$, à la présence unique de la bande lorsque ${ }^{4} \mathrm{~T}_{2}$ se place en dessous de ${ }^{2} \mathrm{E}$.

Ainsi le maximum de la bande ${ }^{4} \mathrm{~T}_{2} \rightarrow{ }^{4} \mathrm{~A}_{2}$ est situé à $705 \mathrm{~nm}$ dans le $\mathrm{YAG}$, à $753 \mathrm{~nm}$ dans GGG $(\mathrm{Ca}, \mathrm{Zr})$ et $760 \mathrm{~nm}$ dans GSGG. Cette situation est favorisée par le fait que ${ }^{4} \mathrm{~A}_{2} \rightarrow{ }^{2} \mathrm{E}$ est une transition presque interdite, ${ }^{4} \mathrm{~A}_{2}$ et ${ }^{2} \mathrm{E}$ appartenant à la même configuration électronique $\mathrm{t}_{2 \mathrm{~g}}$, alors que ${ }^{4} \mathrm{~A}_{2} \rightarrow{ }^{4} \mathrm{~T}_{2}$ est une transition permise non seulement par la règle du spin mais aussi par celle des configurations puisque ${ }^{4} \mathrm{~T}_{2}$ appartient à $\mathrm{e} g$ et que ${ }^{4} \mathrm{~A}_{2}$ appartient à $\mathrm{t} 2 \mathrm{~g}$. Cette bande ${ }^{4} \mathrm{~T}_{2} \rightarrow{ }^{4} \mathrm{~A}_{2}$ est ainsi exploitée dans les grenats comme GGG et GSGG (laser de 740 à $840 \mathrm{~nm}$ ), et dans d'autres réseaux comme l'alexandrite $\mathrm{BeAl}_{2} \mathrm{O}_{4}$ (laser de 700 à $820 \mathrm{~nm}$ ), l'émeraude $\mathrm{Be}_{3} \mathrm{Al}_{2}\left(\mathrm{SiO}_{3}\right)_{6}$ (laser de 730 à $810 \mathrm{~nm}$ ), les gallates mixtes comme $\mathrm{La}_{3} \mathrm{Ga}_{5} \mathrm{GeO}_{14}$ (laser de 880 à $1220 \mathrm{~nm}$ ), les fluorures $\mathrm{KZnF}_{3}$ (laser de 760 à $860 \mathrm{~nm}$ ) ou plus récemment par $\mathrm{LiCaAlF}_{6}$ (laser de 720 à $840 \mathrm{~nm}$ ). Le tableau 1 résume quelques performances des principaux lasers utilisés.

Par cet exemple du chrome on a là une première approche des recherches sur les lasers à solide accordables à la température ambiante. Le paramètre température est extrêmement important puisqu'il conditionne les populations des niveaux vibrationnels des niveaux mis en jeu pour la transition laser ainsi que la compétition entre relaxations non radiatives et radiatives. On comprend donc qu'il existe beaucoup d'autres possibilités aux plus basses températures qui ne sont pas développées pour des raisons technologiques. Cependant, pour des raisons purement fondamentales les études spectroscopiques nécessitent d'étudier les comportements thermiques des centres activateurs à partir de l'hélium liquide afin de mieux maitriser la cinétique totale. La connaissance des principales données spectroscopiques comme la position des niveaux d'absorption et d'émission, la durée de vie des niveaux excités, la largeur de la bande de fluorescence, la section efficace d'émission stimulée sont capitales pour essayer de comprendre les mécanismes de relaxations non radiatives et donc la diminution du rendement de l'émission laser. Ces dernières années, l'attention s'est surtout portée sur les absorptions entre les états excités à partir des niveaux métastables de longue durée de vie qui peuvent réabsorber soit le photon laser, soit le photon de pompage au détriment du rendement global du laser. On a représenté sur la figure 3 ces deux possibilités par des flèches en pointillés dans le cas de l'ion $\mathrm{Cr}^{3+}$. En raison de la forte intensité des sources de pompage, l'absorption entre les états excités est un élément important de la prédiction de nouveaux centres lasers et doit être analysée soigneusement [11].

De nouveaux matériaux lasers accordables dopés $\mathrm{Cr}^{3+}[13]$ sont en cours de développement. Leurs compositions respectives sont :
$\mathrm{LiCaAlF}_{6}: \mathrm{Cr}^{3+} \quad \mathrm{LiCAF}$
LiSrAlF $6: \mathrm{Cr}^{3+} \quad$ LiSAF
LiSrGaAlF $6: \mathrm{Cr}^{3+}$ LiSGAF

Ce groupe de matériaux a été développé à Lawrence Livermore National Laboratory dans le cadre du Programme Laser sur la fusion par confinement inertiel. Ses propriétés spectroscopiques, résumées sur le tableau 2, sont particulièrement intéressantes avec une large bande d'émission donc accordable dans le proche i-r $(700-900 \mathrm{~nm}$ pour LiCAF et $750-1000 \mathrm{~nm}$ pour LiSAF), une longue durée de vie du niveau excité émetteur $(170 \mu$ s pour LiCAF comparé à $3.2 \mu$ s pour le saphir dopé titane) donc facilement pompé par des lampes à éclairs, d'autant 
Tableau 1: Caractéristiques des lasers à solide à la température ambiante

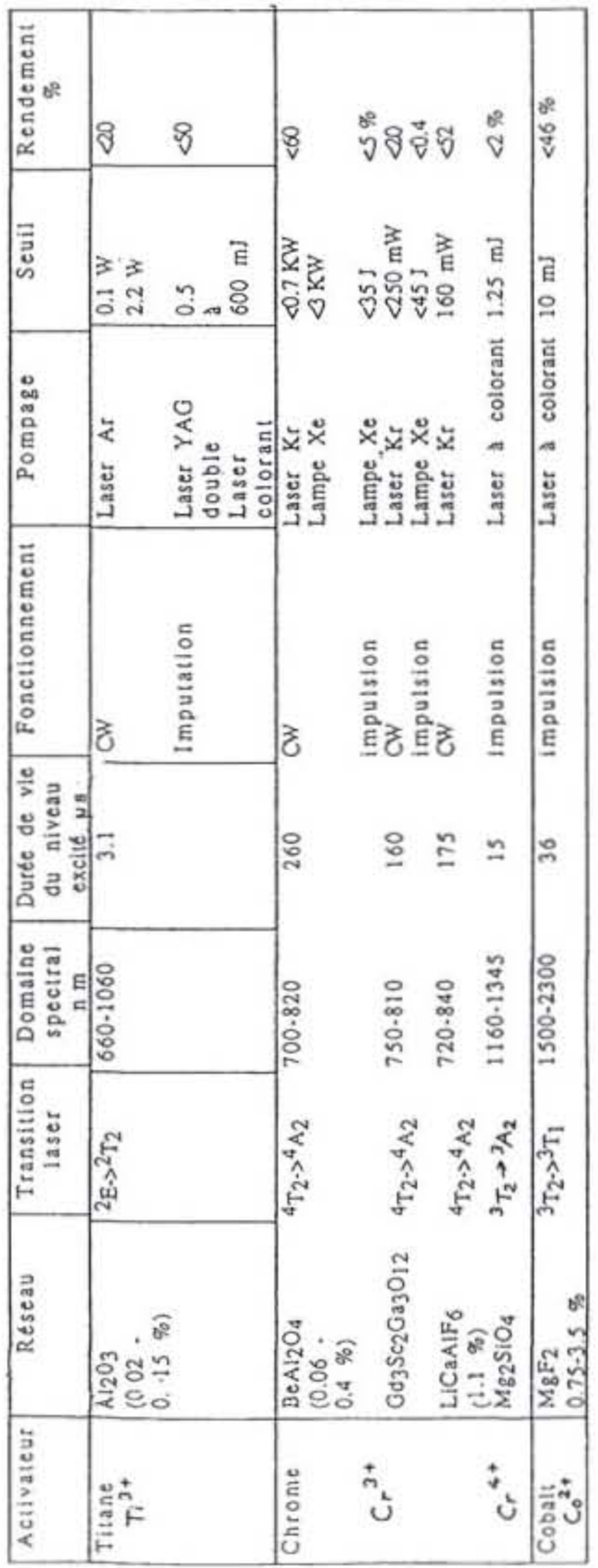


plus que ses bandes d'absorption recouvrent bien le spectre d'émission des lampes au Xe. De plus, l'effet de lentille thermique est faible, l'indice de réfraction non linéaire également et l'absorption dans l'état excité reste négligeable.

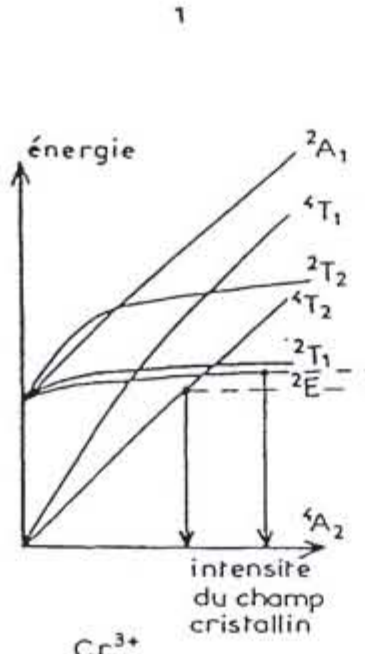

2

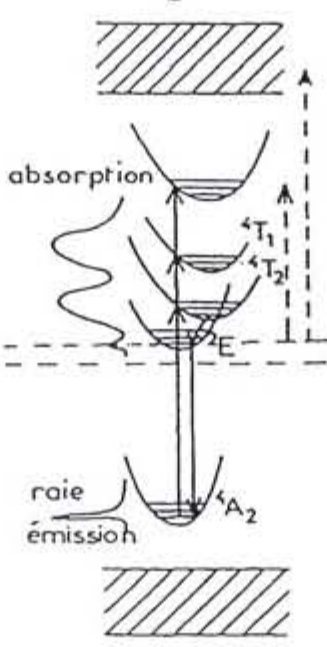

3

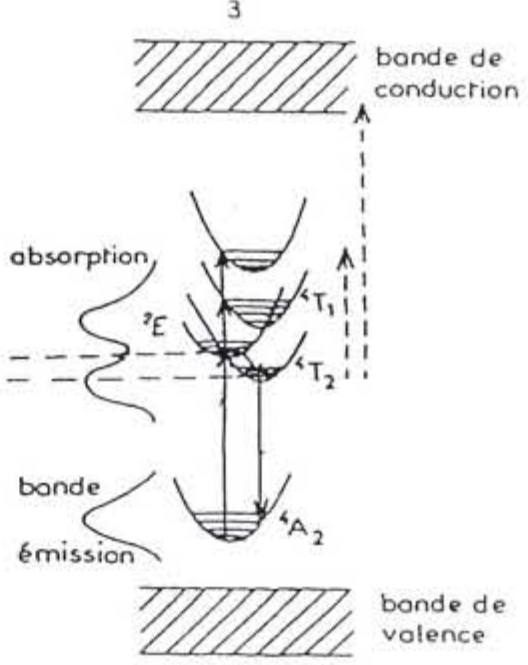

Figure 3 : Diagramme des niveaux d'énergie de Tanabé et Sunago (1) et représentation des courbes de configuration des états excités en champ fort (2) et en champ faible (3). Les spectres d'absorption et d'émission raie ${ }^{2} \mathrm{E} \rightarrow \mathrm{A}_{2}$ pour (2) et bande ${ }^{4} \mathrm{~T}_{2} \rightarrow{ }^{4} \mathrm{~A}_{2}$ pour (3) - ont été dessinés sur la partie gauche des modèles tandis que sur la partie droite on a indiqué les possibilités de réabsorption entre les états excités soit de la transiton d'émission, soit celle de la source de pompage.

Une autre raison, et non la moindre, du succès de ces matériaux est liée à la cristallogénèse du matériau qui accepte des concentrations élevées de chrome avec une distribution uniforme. Ce sont donc des candidats uniques comme lasers accordables et lasers à impulsions courtes à cause de cette bande large, qui peuvent connaître un succès de commercialisation aussi grand que le saphir dopé $\mathrm{Ti}^{3+}$. Signalons à ce propos qu'actuellement le cristal saphir dopé $\mathrm{Ti}^{3+}$ est développé dans une douzaine de systèmes commerciaux et que par analogie avec ce système récemment implanté et bien connu des laséristes, les cristaux LiSAF dopés $\mathrm{Cr}^{3+}$ peuvent être non seulement pompés par des lampes à éclairs au Xe, des lasers $\mathrm{CW}$ à argon ou à impulsions comme YAG-Nd doublés en fréquence $(532 \mathrm{~nm})$ associés soit à des colorants, soit à un autre saphir dopé $\mathrm{Ti}^{3+}$, mais aussi et surtout par des diodes lasers AlGaAs. Des résultats récents [14] ont fournis $10 \mathrm{~mJ}$ par impulsion avec un taux de répétition de $10 \mathrm{~Hz}$ et une durée impulsionnelle de $3 \mathrm{~ns}$ dans la bande spectrale $780-850 \mathrm{~nm}$. On a même atteint des impulsions encore plus courtes, sous pompage par diode-laser, de l'ordre de 25 à $100 \mathrm{ps}$. On doit espérer plus de ce matériau puisque sa largeur de bande autorise des impulsions de l'ordre de 50 femtosecondes. 


\begin{tabular}{|c|c|c|c|c|c|}
\hline & $\mathrm{YAG}: \mathrm{Nd}^{3+}$ & verre : $\mathrm{Nd}^{3+}$ & $\mathrm{Al}_{2} \mathrm{O}_{3}: \mathrm{Ti}^{3+}$ & $\mathrm{LiCAF}: \mathrm{Cr}^{3+}$ & LiSAF : $\mathrm{Cr}^{3+}$ \\
\hline $\begin{array}{l}\text { Domaine } \\
\text { d'aecotdabilite } \\
\text { en } n m\end{array}$ & $1064.1 \pm 0.2$ & $1053 \pm 10$ & $660-1100$ & 700.900 & $750-1000$ \\
\hline $\begin{array}{l}\text { Section efficacite } \\
d^{\prime} \in \text { mission stimulte } \\
\left(\times 10^{-20} \mathrm{~cm}^{2}\right)\end{array}$ & 40 & 4 & 30 & 2.4 & 4.8 \\
\hline $\begin{array}{l}\text { Durte oc vie de l'tat } \\
\text { emetteur en Hs }\end{array}$ & 230 & 350 & 3.2 & 170 & 67 \\
\hline Indice de refraction & 1.82 & 1.51 & 1.76 & 1.39 & 1.4 \\
\hline $\begin{array}{l}\text { Indlec non lintaite } \\
\left(\times 10^{-13} \mathrm{c} \mathrm{Su}\right)\end{array}$ & 2.7 & 1.0 & 1.3 & 0.43 & 0.50 \\
\hline $\begin{array}{l}\text { Diffuslon thermique } \\
\text { en } \mathrm{cm}^{2}, \mathrm{~s}^{-1}\end{array}$ & 0.05 & 0.003 & 0.15 & 0.018 & 0.019 \\
\hline $\mathrm{dn} / \mathrm{dT}\left(\times 10^{-6} /{ }^{\circ} \mathrm{C}\right)$ & 8.9 & -5.5 & 12.6 & $\begin{array}{l}-4.6(\perp c) \\
-4.2(\perp c)\end{array}$ & \\
\hline
\end{tabular}

Tableau 2 : Comparaison des propriétés des nouveaux LiCaF et SiSAF avec celles d'autres matériaux commercialisés

\subsection{L'ion $\mathrm{Ti}^{3+}\left(3 \mathrm{~d}^{1}\right)$}

C'est principalement dans la matrice $\mathrm{Al}_{2} \mathrm{O}_{3}$ que le titane s'est révélé être un ion activateur du plus haut intérêt pour l'instrumentation laser. Les propriétés optiques de ce matériau - on l'appelle le saphir dopé titane - avaient été étudiées dans les années 60 mais ce n'est qu'en 1986 que Moulton décrivaient ses caractéristiques dans une cavité laser à partir d'un monocristal obtenu par la méthode Czochralski [15]. Initialement les échantillons ne donnaient pas entière satisfaction et nous avons alors apporté une contribution en entreprenant une "recherche et développement" d'un laser saphir dopé titane à partir d'une collaboration avec la société Djeva en Suisse et l'Ecole Nationale Supérieure de Chimie de Paris sur des cristaux dont la croissance est obtenue par la méthode de Verneuil [16]. Aujourd'hui ces deux types de lasers à impulsions pompés par un YAG:Nd doublé $(532 \mathrm{~nm})$ sont commercialisés, de même que les lasers continus pompés par un laser à argon $(514,5 \mathrm{~nm})$.

La configuration électronique $3 \mathrm{~d}^{1}$ de l'ion $\mathrm{Ti}^{3+}$ est tout à fait simple puisqu'elle n'implique qu'un seul électron. En coordination octaédrique le niveau fondamental est ${ }^{2} \mathrm{~T}_{2}$ et le niveau excité est ${ }^{2} \mathrm{E}$. La figure 4 représente l'absorption ${ }^{2} \mathrm{~T}_{2} \rightarrow{ }^{2} \mathrm{E}$ dans le visible bien adaptée au pompage par les sources classiques et les lasers, ainsi que l'émission ${ }^{2} \mathrm{E} \rightarrow{ }^{2} \mathrm{~T}_{2}$ assez large dans le rouge et le proche infrarouge permettant l'accordabilité de l'effet laser. Il n'y a pas d'autres niveaux excités supérieurs au-dessus de ${ }^{2} \mathrm{E}$ ce qui élimine d'emblée toute absorption entre les états excités propres au centre $\mathrm{Ti}^{3+}$ contrairement au cas de $\mathrm{Cr}^{3+}$. C'est probablement une raison majeure de la valeur élevée du rendement lumineux du laser mais ce n'est pas la seule car la position des 2 niveaux ${ }^{2} \mathrm{~T}_{2}$ et ${ }^{2} \mathrm{E}$ dans la bande interdite de $\mathrm{Al}_{2} \mathrm{O}_{3}$ (environ $7,5 \mathrm{eV}$ ) doit elle aussi intervenir en excluant toute remontée vers la bande de conduction aussi bien du photon excitateur que du photon laser (voir figure 4). 


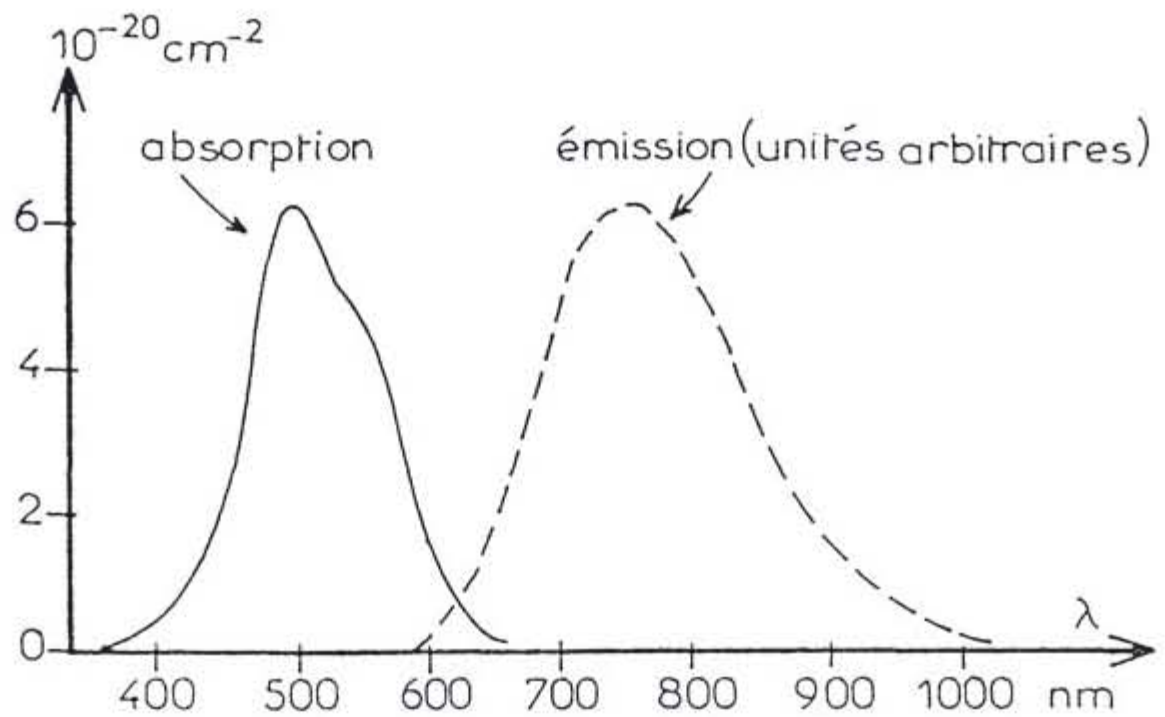

Figure 4 : Spectres d'absorption $\left({ }^{2} \mathrm{~T}_{2} \rightarrow{ }^{2} \mathrm{E}\right)$ et d'émission $\left({ }^{2} \mathrm{E} \rightarrow{ }^{2} \mathrm{~T}_{2}\right)$ du saphir dopé titane. La constante de temps du niveau excité ${ }^{2}$ E est de $3.1 \mu$ s.

La recherche de nouveaux matériaux dopés par $\mathrm{Ti}^{3+}$ a montré que ce n'était pas général et que, par exemple, avec la pérovskite $\mathrm{YAlO}_{3}$, il était beaucoup plus difficile d'obtenir l'émission laser, l'absorption entre les états excités étant beaucoup plus forte coïncidant parfaitement avec l'absorption fondamentale pour $\lambda<250 \mathrm{~nm}$ [17].

En fait, la limitation la plus importante du rendement du laser saphir dopé titane est plutôt liée à la croissance cristalline des échantillons soit par Czockralski, soit par Verneuil qui contiennent tous des traces de paires stables $\mathrm{Ti}^{3+}-\mathrm{Ti}^{4+}$ donnant lieu à une bande de parasite d'absorption vers le rouge orangé en coïncidence avec le début de l'émission de $\mathrm{Ti}^{3+}$.

\subsection{Autres ions}

Enfin, mentionnons que les efforts sur les ions $\mathrm{V}^{2+}, \mathrm{Co}^{2+}$ et $\mathrm{Ni}^{2+}$, ont commencé de porter leur fruit, en particulier pour $\mathrm{MgF}_{2}: \mathrm{Co}^{2+}\left({ }^{3} \mathrm{~d}^{7}\right)$ accordable de 1,5 à $2,3 \mu \mathrm{m}$ qui atteint le stade de la commercialisation. De plus, d'autres ions sont en cours d'analyse dans les laboratoires comme $\mathrm{V}^{4+}, \mathrm{Mn}^{4+}, \mathrm{Mo}^{3+}, \mathrm{Cr}^{4+}, \mathrm{Mn}^{5+}$ ou $\mathrm{Cu}^{+}$et $\mathrm{Ag}^{+}$mais restent encore au stade de la prospection à l'exception de $\mathrm{Cr}^{4+}\left({ }^{3} \mathrm{~d}^{2}\right)$ en symétrie tétraédrique dans la forstérite $\mathrm{Mg}_{2} \mathrm{SiO}_{4}$ et le YAG qui sont utilisés comme cristaux lasers accordables dans le proche infrarouge (figures 5) ou comme absorbant saturable pour les lasers YAG (Nd) à $1,064 \mu \mathrm{m}$. (voir figure $2 \mathrm{~b}$ ). 


\section{LES IONS DE TERRES RARES TRIVALENTES COMME IONS ACTIFS}

Depuis la découverte du laser YAG: $\mathrm{Nd}^{3+}$ en 1964 par Geusic une recherche intense a été consacrée d'abord au néodyme et ensuite à d'autres terres rares comme $\mathrm{Pr}^{3+}, \mathrm{Ho}^{3+}, \mathrm{Er}^{3+}$, $\mathrm{Tm}^{3+}, \mathrm{Yb}^{3+}$ pour ne citer que celles qui débouchent réellement sur des réalisations concrètes de nouvelles cavités lasers. Les principales transitions lasers ont été résumées sur la figure 6 pour les ions $\mathrm{Nd}^{3+}, \mathrm{Ho}^{3+}, \mathrm{Er}^{3+}$ et $\mathrm{Tm}^{3+}$.

\subsection{L'ion $\mathrm{Nd}^{3+}$}

Le succès de l'ion $\mathrm{Nd}^{3+}$ est lié à la possibilité de pomper la multitude des niveaux excités dans le visible suivi d'une relaxation rapide vers le niveau ${ }^{4} \mathrm{~F}_{3 / 2}$ puis de l'émission laser la plus connue vers $1.064 \mu \mathrm{m}$ par la transition $4 \mathrm{~F}_{3 / 2} \rightarrow{ }^{4} \mathrm{I}_{11 / 2}$ et enfin d'une autre relaxation rapide vers le niveau fondamental ${ }^{4} I_{9 / 2}$ (figure 7). L'écart de $2000 \mathrm{~cm}^{-1}$ entre ${ }^{4} I_{9 / 2}$ et ${ }^{4} I_{11 / 2}$ représente environ $10 \mathrm{kT}$ ( $\mathrm{k}$ est la constante de Boltzmann et $\mathrm{T}$ la température absolue) est bien adapté au bon fonctionnement d'un système à 4 niveaux tel qu'il est représenté sur la figure 1 ce qui rend l'observation de la transition laser relativement facile à partir d'un seuil d'énergie faible. Il est curieux de constater que depuis les années 60, et malgré des efforts importants, YAG:Nd n'a pas pu être détrôné en raison certes de ses excellentes propriétés optiques, mais aussi de ses propriétés thermomécaniques remarquables.

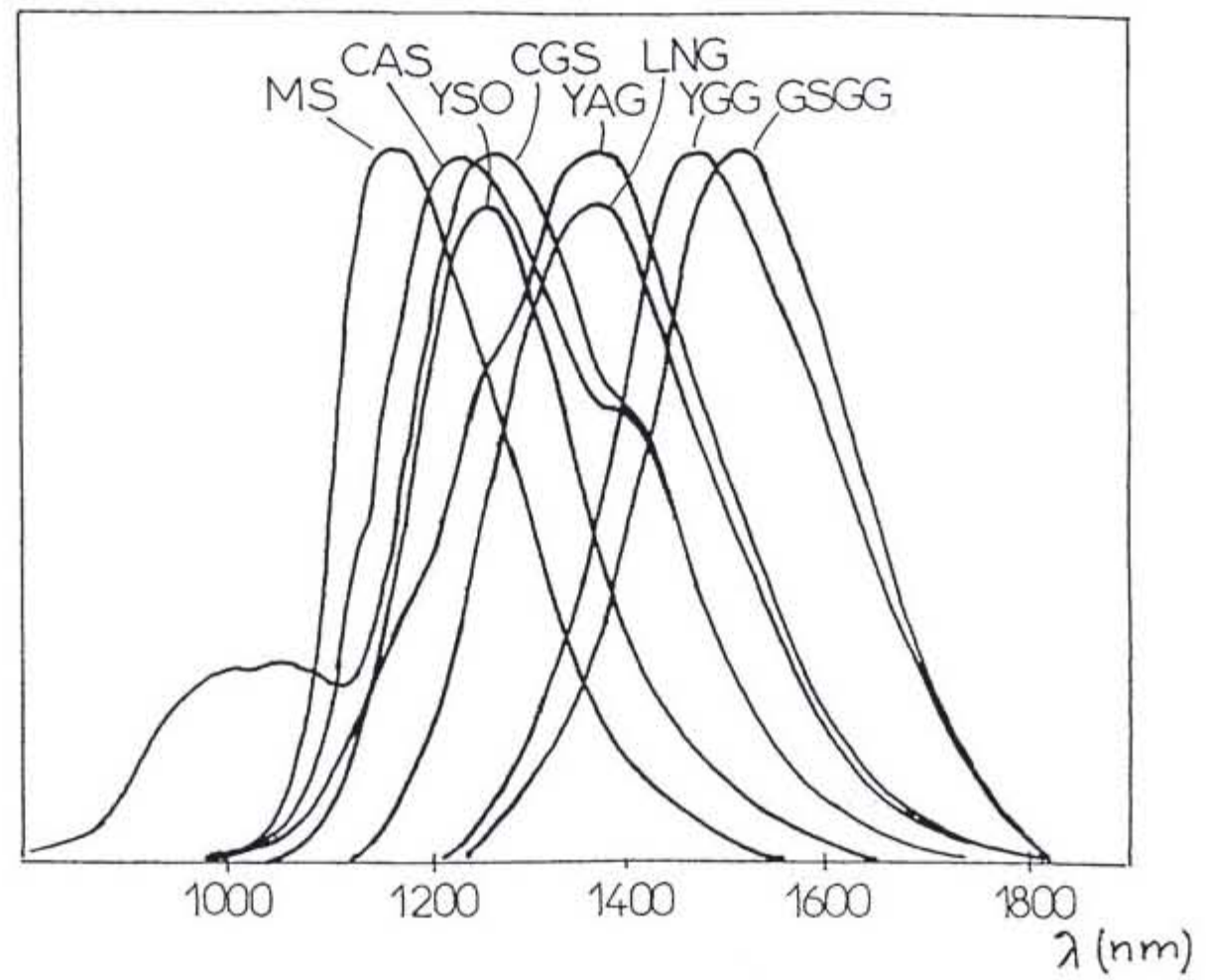

Figure 5 a : Spectre d'émission de cristaux dopés par l'ion $\mathrm{Cr}^{4+}$ en site de symétrie tétraédrique $\mathrm{MS}: \mathrm{Mg}_{2} \mathrm{SiO}_{4}$ - CAS: $\mathrm{Ca}_{2} \mathrm{Al}_{2} \mathrm{SiO}_{7}$ - YSO: $\mathrm{Y}_{2} \mathrm{SiO}_{5}$ - ING:LiNbGeO

$\mathrm{CGS}: \mathrm{Ca}_{2} \mathrm{Ga}_{2} \mathrm{SiO}_{7}$

$\mathrm{YAG}: \mathrm{Y}_{3} \mathrm{Al}_{5} \mathrm{O}_{12}$ - YGG:Y $\mathrm{YGa}_{5} \mathrm{O}_{12}$ - GSGG: $\mathrm{Gd}_{3} \mathrm{Sc}_{2} \mathrm{Ga}_{3} \mathrm{O}_{12}$ 


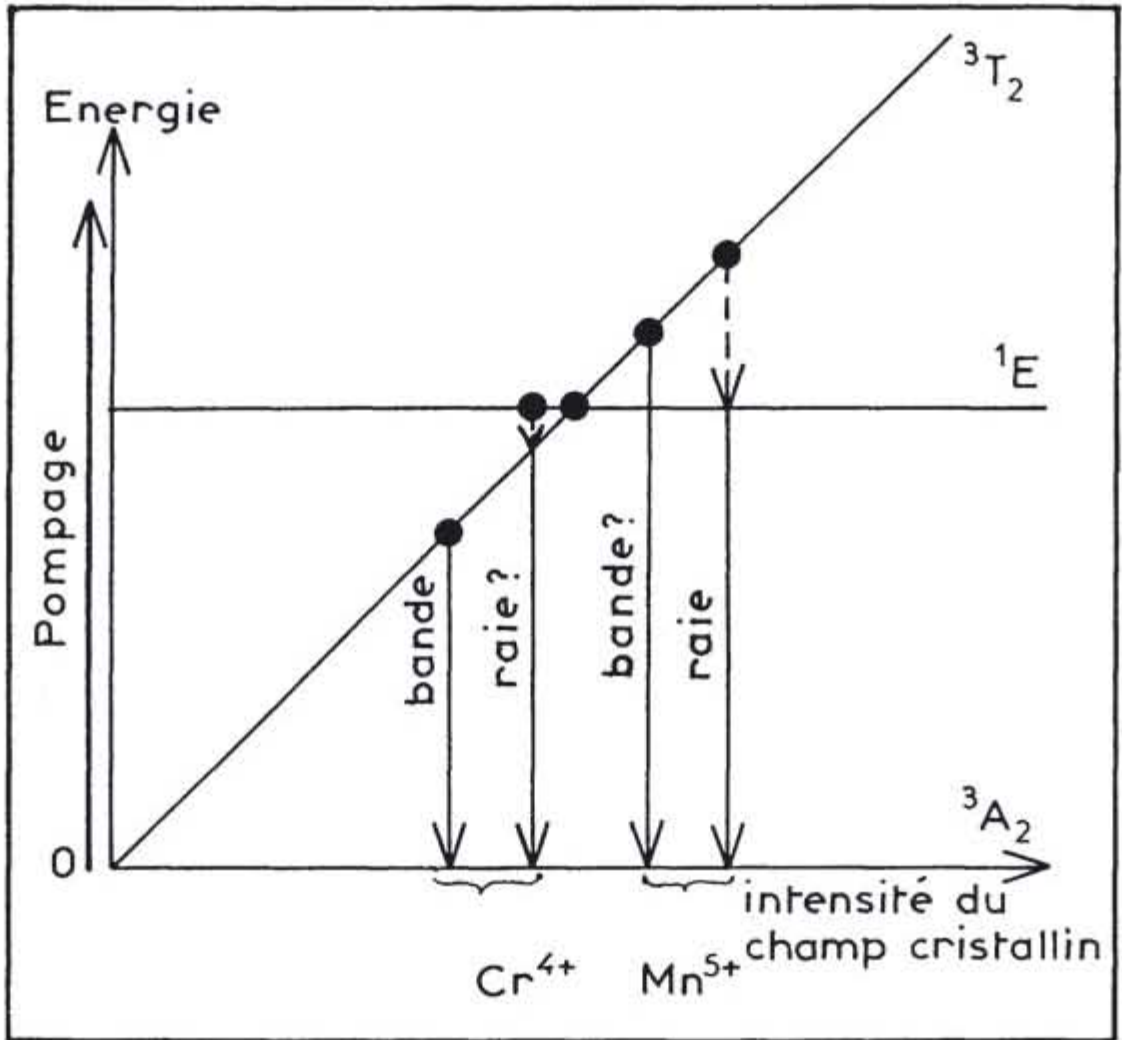

Figure 5 b : Diagramme simplifié de Tanabé et Sugano pour le niveau fondamental ${ }^{3} \mathrm{~A}_{2}$ et les deux premiers niveaux excités ${ }^{1} \mathrm{E}$ et ${ }^{3} \mathrm{~T}_{2}$ des ions $\mathrm{Cr}^{4+}$ et $\mathrm{Mn}^{5+}$ de configuration $3 \mathrm{~d}^{2}$. Compte tenu de l'intensité du champ cristallin représenté en abscisse la tendance de l'ion $\mathrm{Cr}^{4+}$ est plutôt d'émettre la bande large ${ }^{3} \mathrm{~T}_{2} \longrightarrow{ }^{3} \mathrm{~A}_{2}$ en champ faible alors que l'ion $\mathrm{Mn}^{5}+$ émet plutôt la raie ${ }^{1} \mathrm{E} \longrightarrow 3 \mathrm{~A} 2$ en champ fort après pompage sur les niveaux excités élevés et relaxation non-radiative respectivement vers ${ }^{3} \mathrm{~T}_{2}$ et ${ }^{1} \mathrm{E}$. Pour certains cristaux, on peut rencontrer un mélange de la raie et de la bande, celle-ci pouvant aussi être accompagnée de sa raie à O-phonon.

Par exemple, on doit relever aujourd'hui que les cristaux de GSGG codopés par $\mathrm{Cr}^{3+}$ et $\mathrm{Nd}^{3+}$ donnent un rendement deux fois supérieur à celui de YAG:Nd lequel est uniquement de l'ordre de 1 à $2 \%$ dans les cristaux commercialisés mais présente toutefois des propriétés thermiques inférieures sous le pompage des lampes à éclairs au xénon ce qui limite leur développement. Une raison du succès de ce matériau laser peut être lié à l'absence d'absorption entre les états excités aussi bien pour $\lambda=1,064 \mu \mathrm{m}$ que pour $\lambda$ autour de $1,320 \mu \mathrm{m}$ (voir 
figures 8,9 et 10) [18].

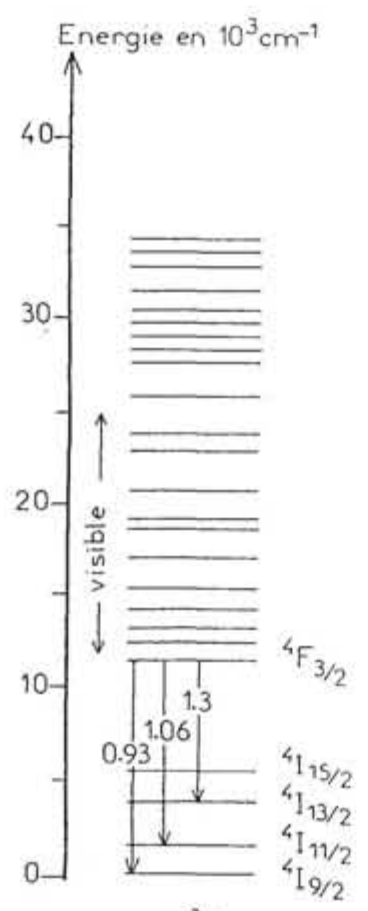

$\mathrm{Nd}^{3+}$

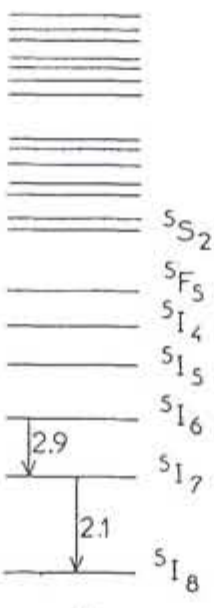

$\mathrm{Ho}^{3+}$

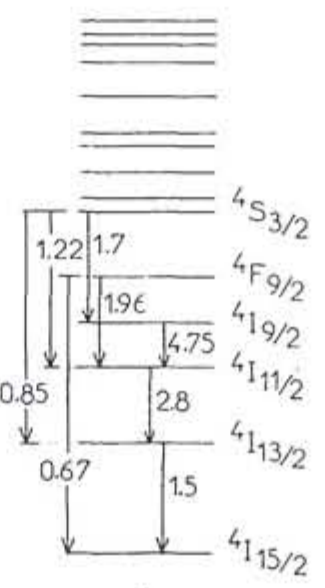

$\mathrm{Er}^{3+}$
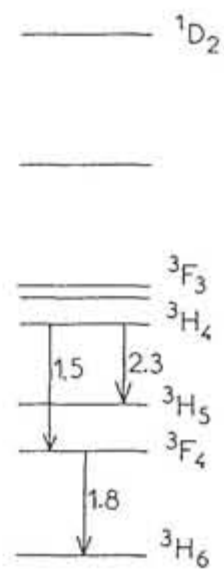

$\mathrm{Tm}^{3+}$

Figure 6: niveaux d'énergie et longueurs d'onde des émissions lasers en $\mu \mathrm{m}$ pour les ions terres rares $\mathrm{Nd}^{3+}, \mathrm{Ho}^{3+}, \mathrm{Er}^{3+}$ et $\mathrm{Tm}^{3+}$

Les cristaux de YAG:Nd ${ }^{3+}$ peuvent émettre deux autres longueurs d'onde à $1317 \mathrm{~nm}$ et 946 $\mathrm{nm}$ qui sont aussi utiles pour quelques applications et bien sûr n'oublions pas le doublage (532 $\mathrm{nm})$, le triplage $(355 \mathrm{~nm})$ et le quadruplage $(266 \mathrm{~nm})$ de fréquence du faisceau à $1064 \mathrm{~nm}$ qui sont des harmoniques couramment commercialisés grâce à l'utilisation des cristaux non linéaires tels que KTP et BBO. En fait, si l'on s'en tient à la valeur absolue du rendement du laser la meilleure approche consiste à pomper le cristal de YAG:Nd ${ }^{3+}$ par des diodes lasers du type GaAlAs/Ga As émettant vers $808 \mathrm{~nm}$, maximum d'absorption du néodyme dans cette région spectrale (figure 7). Ces nouveaux systèmes lasers sont en pleine expansion [19].

Parmi les nouveaux matériaux lasers susceptibles de concurrencer YAG: ${ }^{3}{ }^{3+}$, citons :

- $\mathrm{LiYF}_{4}: \mathrm{Nd}^{3+}$ seul réseau caractérisé par une variation négative de l'indice de réfraction en fonction de la température évitant ainsi le phénomène de focalisation thermique [20]. La longueur d'onde $\lambda=1.053 \mu \mathrm{m}$ est utilisée pour les oscillateurs de l'expérience de fusion par confinement inertiel. Ce cristal est également caractérisé par une faible influence de l'absorption entre les états excités (voir figure 9) [18].

- $\mathrm{LaMgAl} \mathrm{Al}_{11} \mathrm{O}_{19}: \mathrm{Nd}^{3+}$, composé uniaxial émettant aussi bien à $1,055 \mu \mathrm{m}$ qu'à $1,081 \mu \mathrm{m}$ qui est utilisé pour les expériences de pompage de l'hélium 3 polarisé. L'extinction par concentration est réduit car il peut contenir des teneurs supérieures à celles de YAG qui est, rappelons le, limité à 1 atome \%. De plus, sa cristallogénèse est favorisée par un coefficient de 
ségrégation voisin de l'unité [21].

- $\mathrm{YVO}_{4}: \mathrm{Nd}^{3+}$ pour la position du niveau ${ }^{4} \mathrm{~F}_{3 / 2}$ bien adapté au pompage des diodes lasers en raison de sa très large bande qui serait homogène.

- Les grenats $\mathrm{A}_{3} \mathrm{~B}_{2} \mathrm{C}_{3} \mathrm{O}_{12}$ sont largement étudiés avec $\mathrm{Gd}_{3} \mathrm{Sc}_{2} \mathrm{Ga}_{3} \mathrm{O}_{12}, \mathrm{Y}_{3} \mathrm{Sc}_{2} \mathrm{Ga}_{3} \mathrm{O}_{12}$, $\mathrm{Gd}_{3} \mathrm{Ga}_{5} \mathrm{O}_{12}$ ou encore $(\mathrm{Gd}, \mathrm{Ca})_{3}(\mathrm{Ga}, \mathrm{Mg}, \mathrm{Zr})_{5} \mathrm{O}_{12}$ sans le scandium rare et cher. Le site octaédrique à champ faible peut accepter l'ion $\mathrm{Cr}^{3+}$ comme sensibilisateur dont la bande large d'émission ${ }^{4} \mathrm{~T}_{2} \rightarrow{ }^{4} \mathrm{~A}_{2}$ recouvre plusieurs niveaux excités de $\mathrm{Nd}^{3+}$ et de $\mathrm{Tm}^{3+}$ favorisant les transferts d'énergie radiatifs et non radiatifs entre $\mathrm{Cr}^{3+}$ et $\mathrm{Nd}^{3+}$ (Figure 11) ou $\mathrm{Cr} 3+$ et $\mathrm{Tm}^{3+}$.

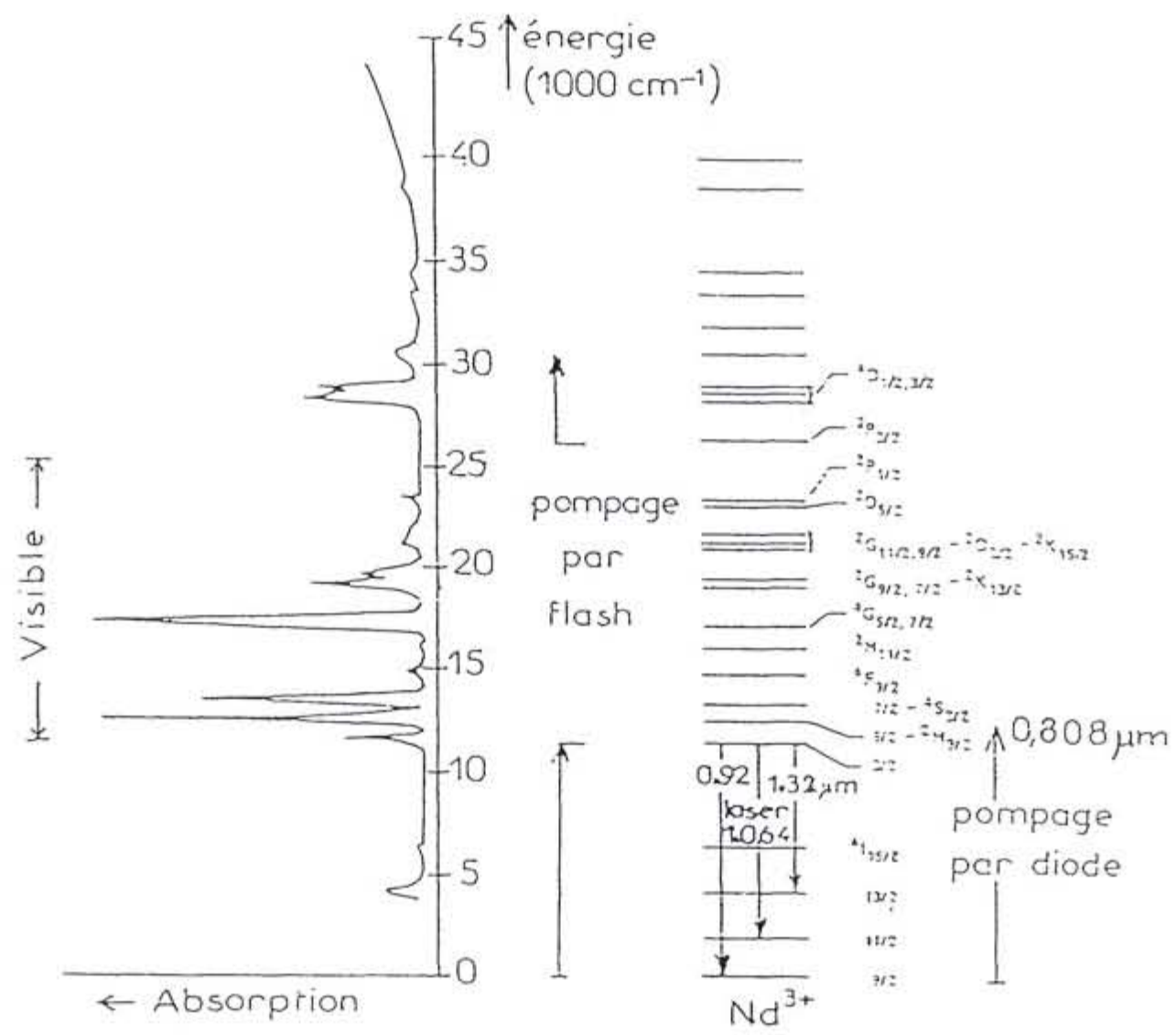

Figure 7: Niveaux d'énergie de l'ion terre rare $\mathrm{Nd}^{3+}$ dans le matériau monocristallin YAG. Le spectre d'absorption permet de comprendre l'utilisation du pompage optique par lampes flashs dans le visible. 3 transitions lasers peuvent être obtenues mais la plus intense correspond à $\lambda=1,064 \mu \mathrm{m}$. Compte tenu de l'association des transitions avec la couche interne $4 \mathrm{f}^{\mathrm{n}}(\mathrm{n}=1$ à 14$)$ des terres rares les raies d'absorption et d'émission ne sont que légèrement déplacées selon l'environnement cristallin quand on change le réseau hôte. On a aussi précisé le pompage par diode -laser autour de $0,808 \mu \mathrm{m}$. 

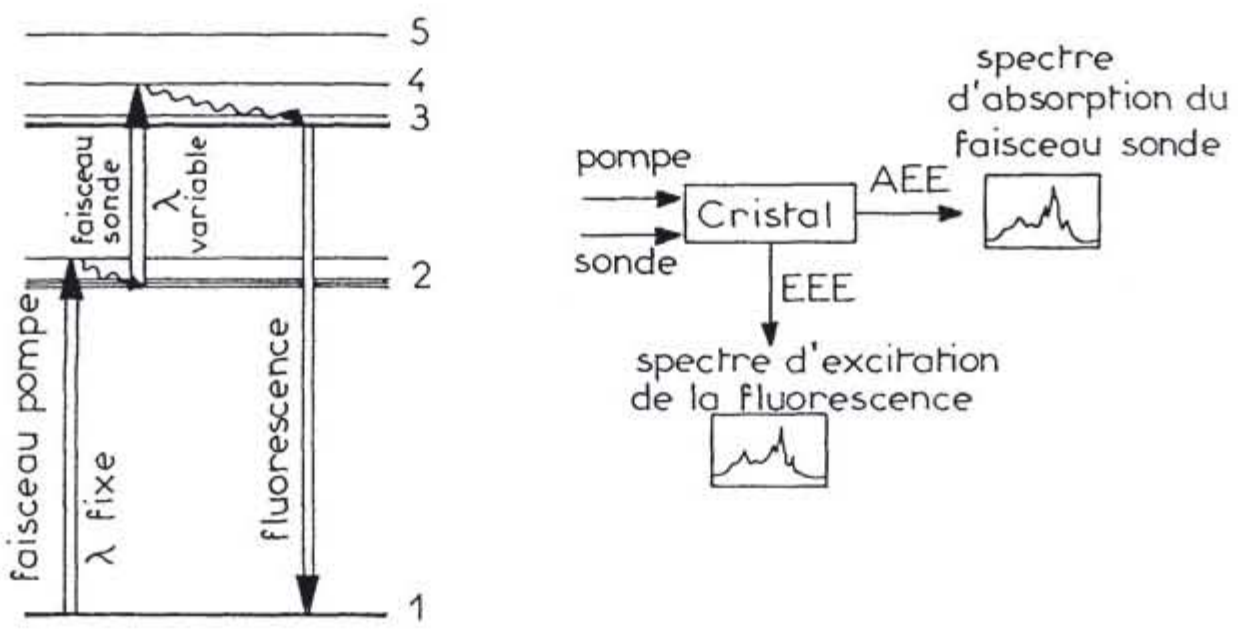

Figure 8: Schéma de principe d'enregistrement des spectres d'absorbtion dans l'état excité (AEE) et des spectres d'excitation dans l'état excité (EEE). La variation de la longueur d'onde $\lambda$ du faisceau sonde permet d'obtenir soit directement le spectre d'AEE entre le niveau (2) et les niveaux (3), (4) et (5), soit celui d'EEE en détectant la fluorescence issue d'un niveau excité plus élevé que le niveau (2).

Une recherche détaillée a été entreprise dans notre groupe sur ces matrices que l'on tire au moyen d'un four automatisé par la méthode Czochralski [11]. Indiquons que $\mathrm{Gd}_{3} \mathrm{Ga}_{5} \mathrm{O}_{12}: \mathrm{Nd}^{3+}$ est actuellement le meilleur matériau laser sous forme de plaquette [9]. De plus, il est possible d'élargir les raies des grenats désordonnés par l'introduction de cations $\mathrm{Ca}^{2+}, \mathrm{Zr}^{4+}$ ou $\mathrm{Nb}^{5+}$ dans $(\mathrm{Gd}, \mathrm{Ca})_{3} \mathrm{Ga}_{2}(\mathrm{Ga}, \mathrm{Zr})_{3} \mathrm{O}_{12}$ et $\mathrm{Ca}_{3}(\mathrm{Nb}, \mathrm{Ga})_{2} \mathrm{Ga}_{3} \mathrm{O}_{12}$ qui ont une bonne conductibilité thermique, qui peuvent être, en outre, tirés avec une excellente qualité optique et qui sont donc proposés pour être pompés par des diodes-lasers.

- Les perovskites comme YAlO 3 (YAP:Nd ${ }^{3+}$ ) dont l'émission est polarisée ou encore un nouveau composé $(\mathrm{La}, \mathrm{Sr})(\mathrm{Al}, \mathrm{Ta}) \mathrm{O}_{3}$ dont l'élargissement inhomogène causé par la distribution des cations est avantageux pour le pompage par diode laser autour de $800 \mathrm{~nm}$ (Figure 12a et 12b) [22].

- Verres de phosphates : $\mathrm{Nd}^{3+}$ sont aussi développés mais ils souffrent, cependant, malgré leur taille importante, d'une mauvaise conductibilité thermique et leur utilisation reste uniquement concentrée sur les amplificateurs des chaînes de laser pour la fusion par confinement inertiel. Trois montages sont connus :

aux États-Unis (lasers NOVA de Lawrence Livermore National laboratory, qui délivre $100 \mathrm{KJ})$,

. au Japon (laser GEKKO XII qui délivre $30 \mathrm{KJ}$ ),

. en France au CEA à Limeil-Valenton (laser Phébus de $20 \mathrm{KJ}$ ). 

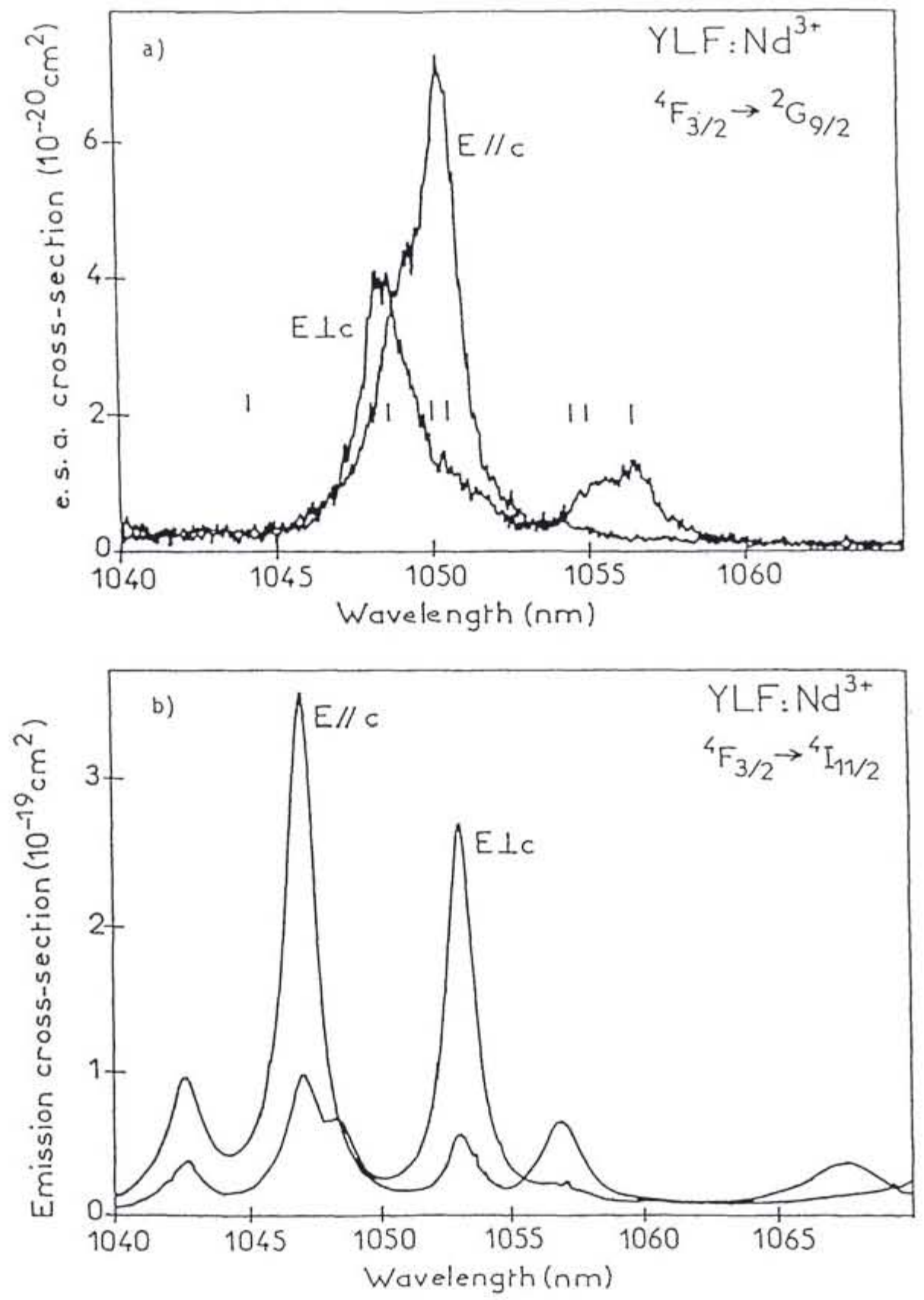

Figure 9 : spectres de sections efficaces a) d'AEE et b) d'émission autour de 1,06 $\mu \mathrm{m}$, enregistrés en lumière polarisée, dans le YLF:Nd ${ }^{3+}$. 

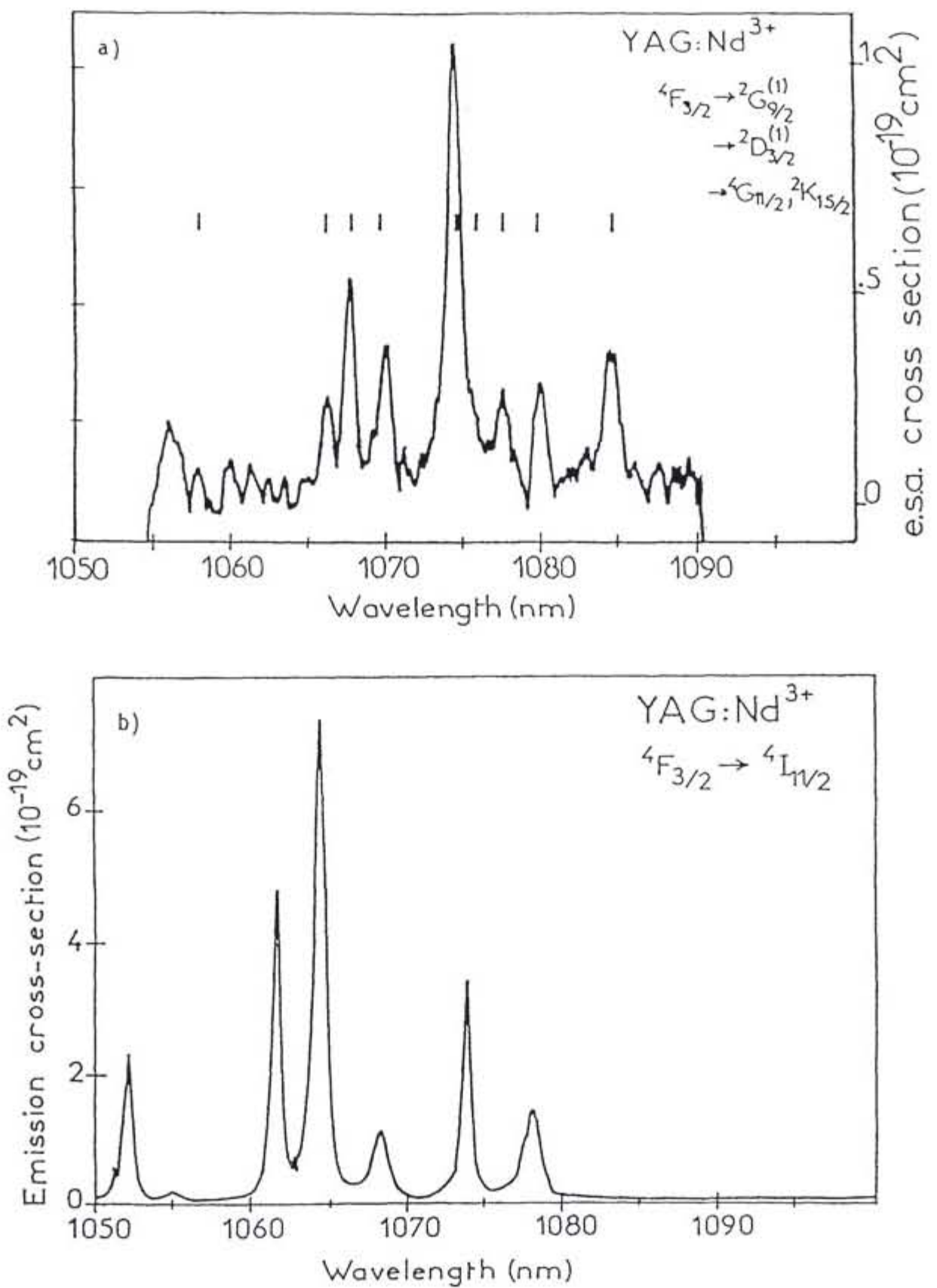

Figure 10 : Spectres de sections efficaces: a) d'AEE et b) d'émission autour de 1,06 $\mu \mathrm{m}$ dans le YAG:Nd ${ }^{3+}$. 


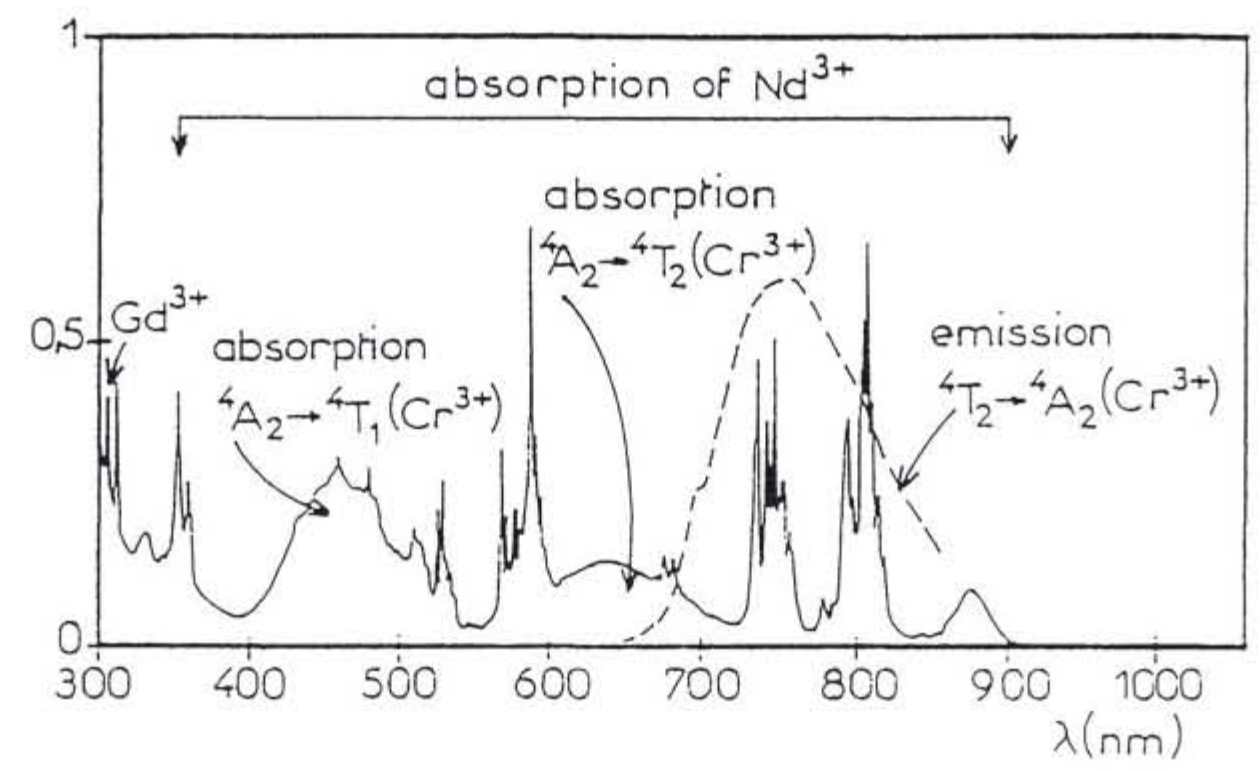

Figure 11 : Spectres d'absorption de $\mathrm{Cr}^{3+}$ et de $\mathrm{Nd}^{3+}$ dans le grenat substitué $\mathrm{GGG}(\mathrm{Ca}, \mathrm{Zr}$ ) (en traits pleins) et spectre d'émission de $\mathrm{Cr}^{3+}$ (en pointillés) à température ambiante. Les ordonnées sont en unités arbitraires. Le transfert est aussi favorisé par les valeurs des durées de vie des niveaux excités ${ }^{4} \mathrm{~T}_{2}$ de $\mathrm{Cr}^{3+}(\tau=$ $110 \mu \mathrm{s})$ et ${ }^{4} \mathrm{~F}_{3 / 2}$ de $\mathrm{Nd}^{3+}(\tau=220 \mu \mathrm{s})$.

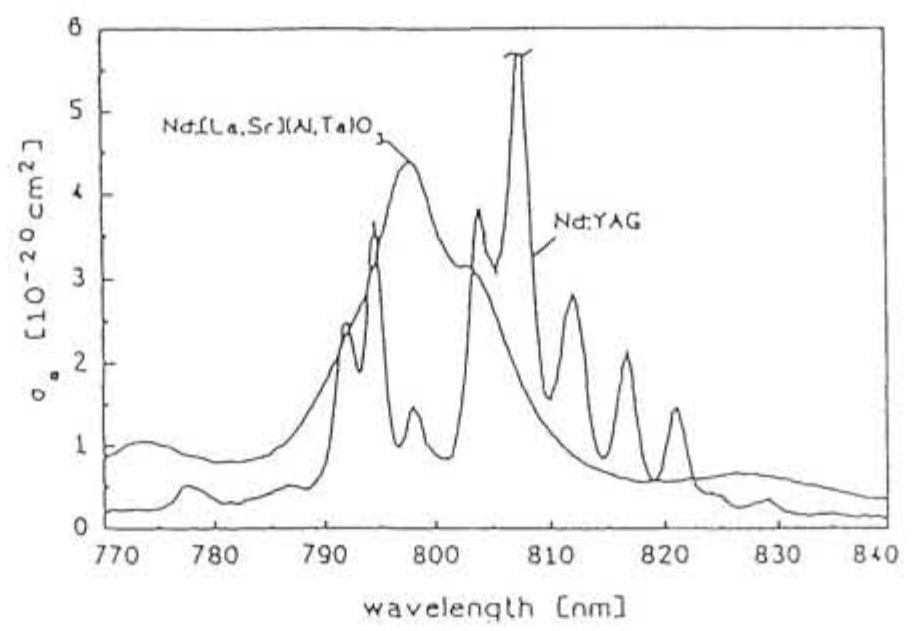

Figure 12a: Spectre d'absorption de Nd:YAG et Nd:[La,Sr] $(\mathrm{Al}, \mathrm{Ta}) \mathrm{O}_{3}$ dans le domaine autour de $800 \mathrm{~nm}$ 


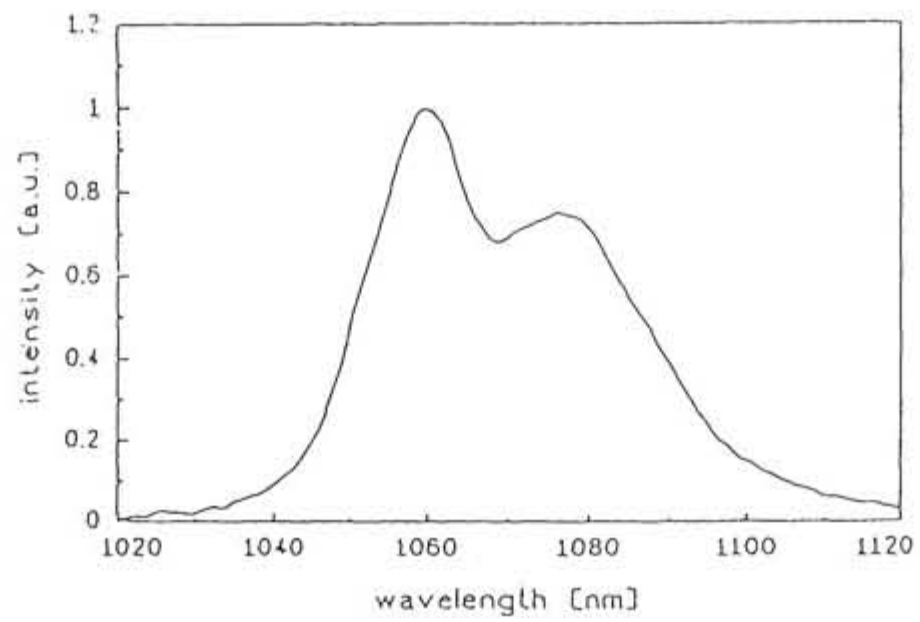

Figure 12b : Spectre d'émission de la transition ${ }^{4} \mathrm{~F}_{3 / 2} \rightarrow{ }^{4} \mathrm{I}_{11 / 2}$ du cristal $\mathrm{Nd}:(\mathrm{La}, \mathrm{Sr})(\mathrm{Al}, \mathrm{Ta}) \mathrm{O}_{3}$.

Les amplificateurs travaillent à la limite du supportable et au CEA les disques elliptiques de verres dopés néodyme, de $46 \mathrm{~cm}$ de longueur, viennent d'être remplacés par des disques octogonaux de $31,5 \mathrm{~cm}$ dans la chaîne du laser Phebus à cause des dégradations irréversibles provoquées par le flux intense du laser. La longueur d'onde de fonctionnement est de $1,05 \mu \mathrm{m}$ à partir d'un oscillateur constitué, non pas d'un YAG, mais d'un cristal LiYF 4 dopé au néodyme dont la longueur d'onde est mieux adaptée à l'émission des amplificateurs. Ce sont d'ailleurs ces outils qui permettent d'approfondir les processus d'interaction laser-matière avec, comme conséquence récente, l'observation de l'émission stimulée dans un plasma de sélénium produit par Phebus aux longueurs d'onde de 206 et $209 \AA$ donc à la conception des lasers à rayons $\mathrm{X}$ qui, on le conçoit, élargiront le champ de nos connaissances dans ce domaine de première importance pour les matériaux.

\subsection{Autres terres rares : $\mathrm{Er}^{3+}, \mathrm{Tm}^{3+}, \mathrm{Ho}^{3+}$}

Ce sont les trois ions les plus étudiés pour leurs différentes émissions lasers observées et exploitées dans le proche infrarouge. La figure 5 précise les principales transitions intéressant les applications aussi diverses que la médecine, l'instrumentation scientifique, le LIDAR (radar lumineux), les capteurs optiques, les communications optiques, les fibres optiques amplificatrices, les télémètres ou encore les lasers infrarouges sans danger pour l'oeil. Les développements les plus poussés concernent le pompage de ces matériaux par des diodes lasers vers $808 \mathrm{~nm}$ avec des possibilités de doublage de fréquence et de conversion de l'excitation vers les niveaux excités supérieurs impliquant un ou plusieurs ions via les transferts d'énergie efficaces.

Avec $\mathrm{Er}^{3+}$ la dynamique des états excités fait intervenir plusieurs processus de relaxation croisée et de conversion vers les états excités supérieurs même en présence d'une faible concentration de l'activateur. Cet ion est aussi un excellent sensibilisateur d'autres ions $\left(\mathrm{Tm}^{3+}\right.$, $\mathrm{Ho}^{3+}$ ) mais peut être également sensibililisé par $\mathrm{Cr}^{3+}$ ou $\mathrm{Yb}^{3+}$.

Les études sur les ions $\mathrm{Tm}^{3+}$ et $\mathrm{Ho}^{3+}$ sont plus récentes. On voit sur la figure 13 que la dynamique des ions $\mathrm{Tm}^{3+}$ est caractérisée par un mécanisme de relaxation croisée efficace du type ${ }^{3} \mathrm{H}_{4} \rightarrow{ }^{3} \mathrm{~F}_{4}$ et ${ }^{3} \mathrm{H}_{6} \rightarrow{ }^{3} \mathrm{~F}_{4}$ entre ions adjacents aussi bien sous pompage directement par 
une diode laser, qu'indirectement par l'intermédiaire de l'ion sensibilisateur $\mathrm{Cr}^{3+}$ qui remplace, avantageusement $\mathrm{Er}^{3+}$ dans les grenats. $\mathrm{Si}$, par ailleurs, les ions $\mathrm{Tm}^{3+}$ sont utilisés à leur tour comme sensibilisateurs de l'émission de $\mathrm{Ho}^{3+}$ à $2,1 \mu \mathrm{m}$, intéressante pour la transmission dans l'une des fenêtres spectrales de l'atmosphère, alors la dissipation de l'énergie thermique dans le cristal décroît considérablement. On peut noter ici la mise en évidence d'un transfert inverse ${ }^{5} \mathrm{I}_{7}\left(\mathrm{Ho}^{3+}\right) \rightarrow{ }^{3} \mathrm{~F}_{4}\left(\mathrm{Tm}^{3+}\right)$ à la température ambiante qui limite à quelques $\%$ la concentration de $\mathrm{Tm}^{3+}$ dans les échantillons optimisés. Avec les grenats on peut augmenter le paramètre de maille $\mathrm{a}_{0}$ en insérant $\mathrm{Ca}^{2+}, \mathrm{Mg}^{2+}$ et $\mathrm{Zr}^{4+}$ afin de réduire le champ cristallin du site octaédrique et donc de favoriser le recouvrement entre la bande ${ }^{4} \mathrm{~T}_{2} \rightarrow{ }^{4} \mathrm{~A}_{2}$ de $\mathrm{Cr}^{3+}$ et les niveaux de $\mathrm{Tm}^{3+}$ et $\mathrm{Ho}^{3+}$ dans une approche similaire à celle des transferts $\mathrm{Cr}^{3+}-\mathrm{Nd}^{3+}$.

Les figures $14 \mathrm{a}$ à $14 \mathrm{e}$ montrent à titre d'exemple l'utilisation de l'ion $\mathrm{Tm}^{3+}$ dans $\mathrm{Y}_{3} \mathrm{~A}_{15} \mathrm{O}_{12}$ ainsi que la comparaison des performances lasers de plusieurs types de cristaux [23].

A cause du nombre élevé de dopants les modèles utilisés pour l'interprétation des transferts sont assez compliqués mais il doit être mentionné que selon la nature soit du réseau (fluorures ou grenats par exemple), soit du sensibilisateur $\left(\mathrm{Cr}^{3+}\right.$ ou $\left.\mathrm{Er}^{3+}\right)$ on fait appel à des régimes différents qui sont d'excellents tests expérimentaux des mécanismes dynamiques entre les états excités. Il est probable que toutes les transitions lasers entre ces ions n'ont pas été exploitées de même pour les transferts d'énergie à partir d'ions de transition différents de $\mathrm{Cr}^{3+}$, comme par exemple le vanadium, le manganèse et d'autres qu'il faudrait développer.

\section{CONCLUSION}

Les recherches sur les matériaux lasers inorganiques dopés par les ions de transitions et par les ions de terres rares sont très actives dans plusieurs laboratoires. L'optimisation des matériaux nécessite un développement accru des fours de tirages du type Czochralski ou Bridgeman de monocristaux de différentes structures à fusion congruente dopés par des teneurs variables d'activateurs et de sensibilisateurs. Compte tenu du nombre élevé de matériaux à tester, on peut maintenant synthétiser des monocristaux sous forme de fibres de petites dimensions par fusion au moyen de lasers $\mathrm{CO}_{2}$, permettant la cristallogénèse rapide d'échantillons dont les points de fusion peuvent dépasser la limite de $1900^{\circ} \mathrm{C}$ imposée par les creusets. Il convient de même d'approfondir les études dynamiques entre les états excités d'un ou plusieurs ions par transfert d'énergie selon la nature de la source de pompage (lampes visibles à éclairs, lasers, diodes lasers). Après s'être assuré de sa potentialité de croissance cristalline sous une forme de barreaux ou de plaquettes, l'aboutissement du développement d'un nouveau matériau dépend bien sûr des ses propriétés optiques mais autant de ses propriétés thermiques et mécaniques. Il n'est donc pas étonnant de ne trouver sur le marché que peu d'entre eux :

Ce sont YAG: $\mathrm{Y}_{3} \mathrm{Al}_{5} \mathrm{O}_{12}: \mathrm{Nd}^{3+}, \mathrm{LiYF}_{4}: \mathrm{Nd}^{3+}, \mathrm{Gd}_{3} \mathrm{Sc}_{2} \mathrm{Ga}_{3} \mathrm{O}_{12}: \mathrm{Cr}^{3+}-\mathrm{Nd}^{3}, \mathrm{Gd}_{3} \mathrm{Ga}_{5} \mathrm{O}_{12}: \mathrm{Nd}^{3+}$, $\mathrm{LaMgAl}{ }_{11} \mathrm{O}_{19}: \mathrm{Nd}^{3+}$, verres : $\mathrm{Nd}^{3+}$, verres : $\mathrm{Er}^{3+}, \mathrm{LYF}_{4}: \mathrm{Er}^{3+}$, rubis : $\mathrm{Al}_{2} \mathrm{O}_{3}: \mathrm{Cr}^{3+}$, alexandrite: $\mathrm{BeAl}_{2} \mathrm{O}_{4}: \mathrm{Cr}^{3+}, \mathrm{MgF}_{2}: \mathrm{Co}^{2+}$, saphir dopé titane : $\mathrm{Al}_{2} \mathrm{O}_{3}: \mathrm{Ti}^{3+}$. Malgré la forte concurrence des cristaux donnant lieu à l'oscillation paramétrique optique $(\mathrm{OPO})$ dans le visible et l'ir on peut penser que l'accroissement des recherches devrait aboutir rapidement à l'émergence d'autres monocristaux aux applications spécifiques pour l'instrumentation scientifique, les lasers industriels, les sources pour le médical, les applications militaires ou encore l'environnement. Parmi les voies les plus novatrices, citons celle de la recherche de matériaux lasers présentant des propriétés non linéaires permettant d'auto-doubler la fréquence de la transition laser habituelle du cristal. On peut aussi mentionner la recherche de matériaux donnant lieu à la conversion de l'énergie ir vers le bleu.

Enfin, on doit rajouter que ces nouveaux matériaux permettent de relancer des recherches fondamentales comme les attributions spectrales relatives aux centres activateurs et à leurs états d'ionisation présents réellement dans le réseau et donc d'approfondir les recherches spectroscopiques comme celles sur la distribution des ions activateurs dans le réseau ou encore celles sur les mécanismes d'interaction entre les dopants à partir de monocristaux d'excellentes qualités optiques. 

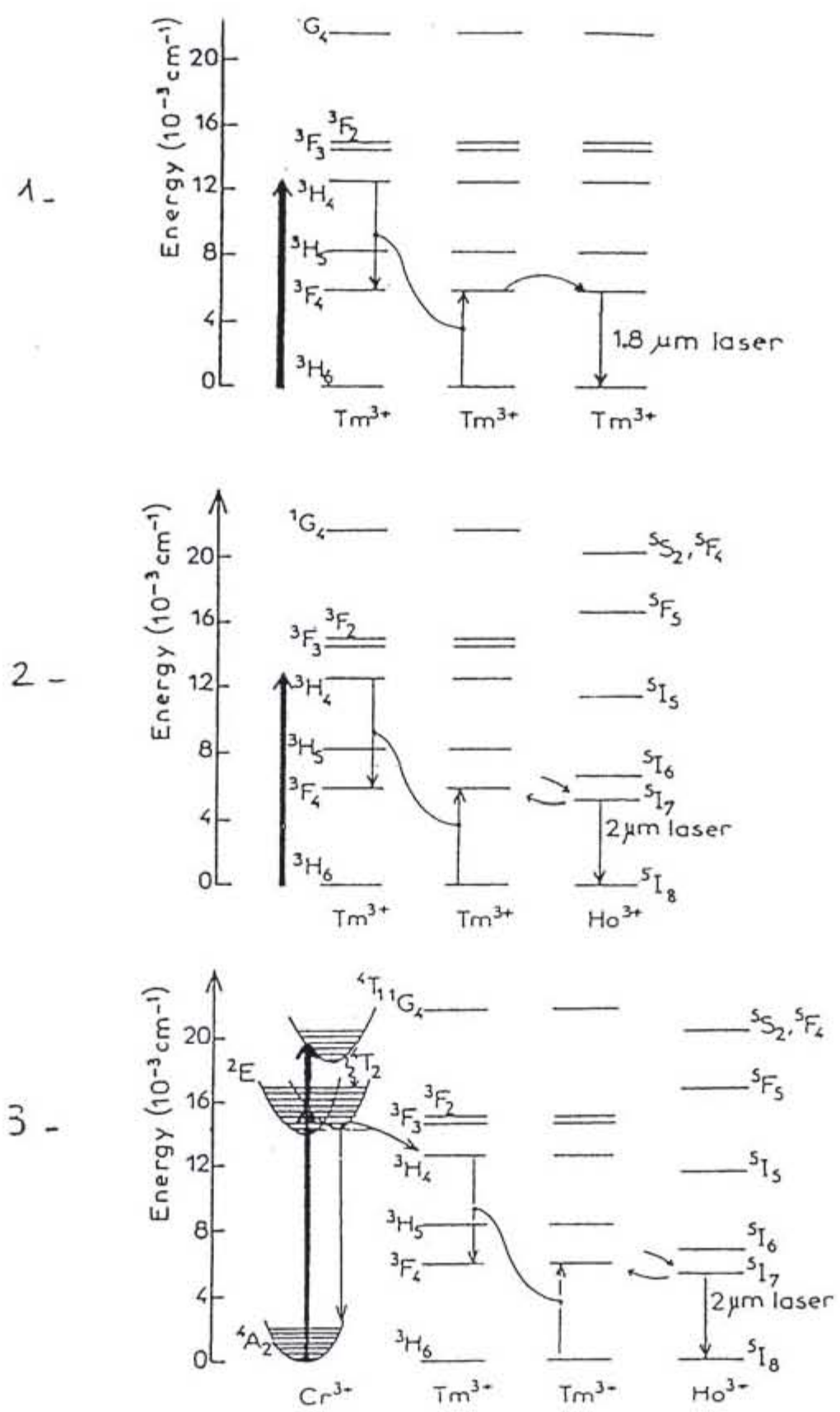

Figure 13 : mécanismes d'absorption et d'émission des ions $\mathrm{Tm}^{3+}(1), \mathrm{Tm}^{3+}-\mathrm{Ho}^{3+}(2)$ et $\mathrm{Cr}^{3+}-\mathrm{Tm}^{3+}$ $-\mathrm{Ho}^{3+}$ (3). La transition ${ }^{3} \mathrm{H}_{6} \rightarrow{ }^{3} \mathrm{H}_{4}$ de $\mathrm{Tm}^{3+}$ est bien adaptée au pompage par diode-laser tandis que les transitions ${ }^{4} \mathrm{~A}_{2} \rightarrow{ }^{4} \mathrm{~T}_{1},{ }^{4} \mathrm{~T}_{2}$ de $\mathrm{Cr}^{3+}$ sont adaptés au pompage par des lampes et des lasers dans le visible. 


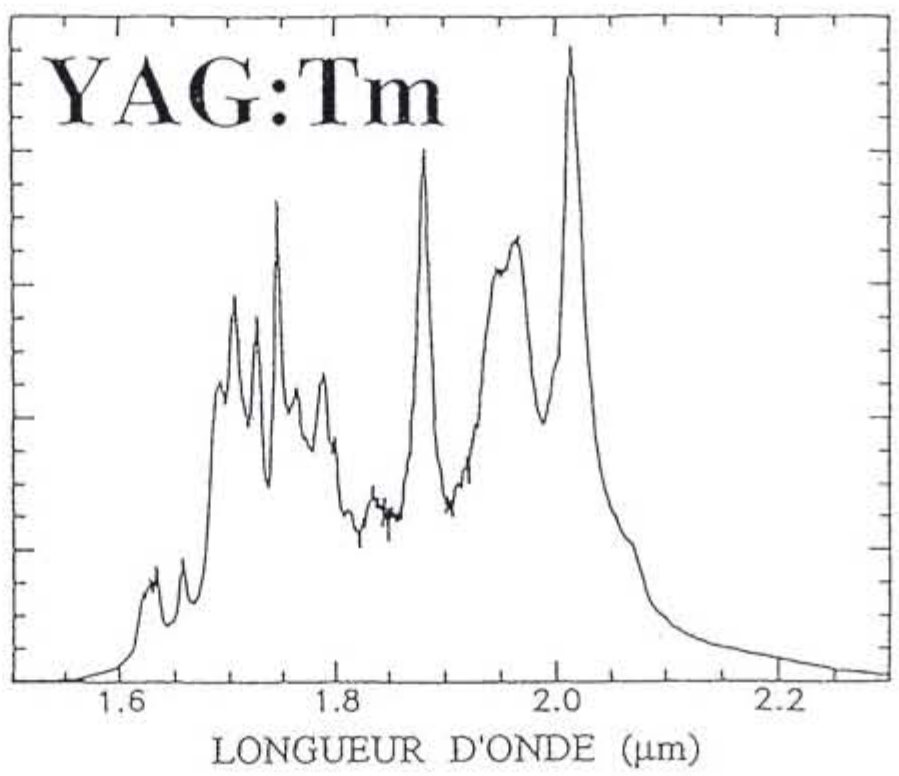

Figure 14a : Spectre d'émission de l'ion $\mathrm{Tm}^{3+}$ dans $\mathrm{Y}_{3} \mathrm{Al}_{5} \mathrm{O}_{12}$ à la température ambiante.

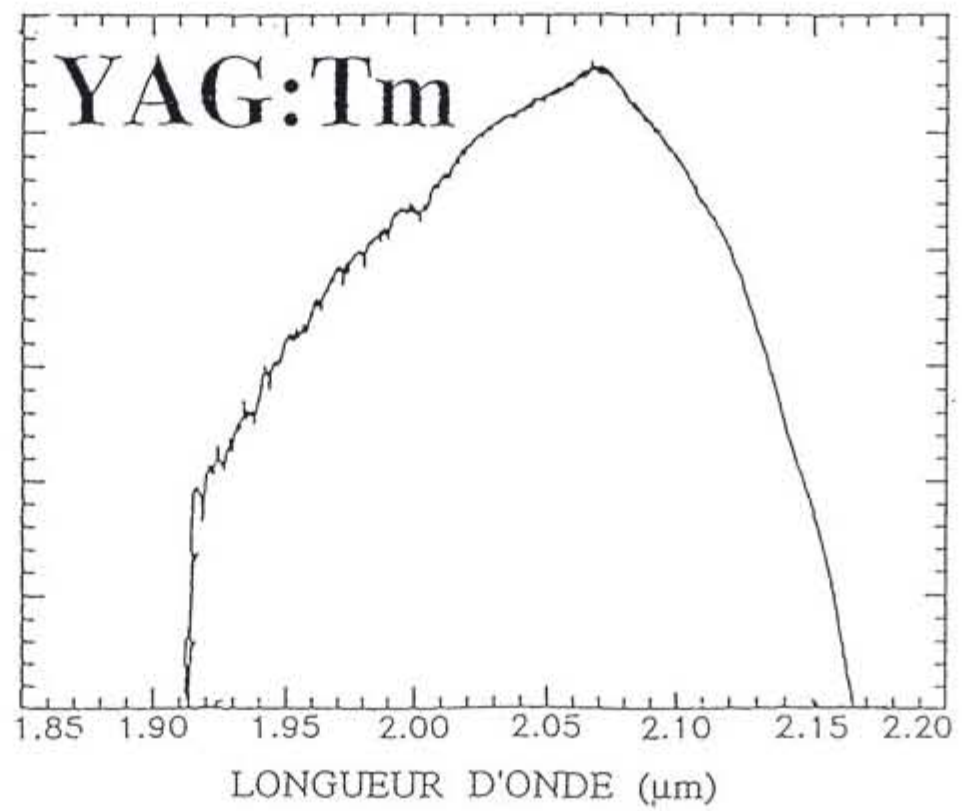

Figure 14b: Domaine d'accordabilité de l'émission laser dans $\mathrm{Y}_{3} \mathrm{Al}_{5} \mathrm{O}_{12}: \mathrm{Tm}^{3+}$. 


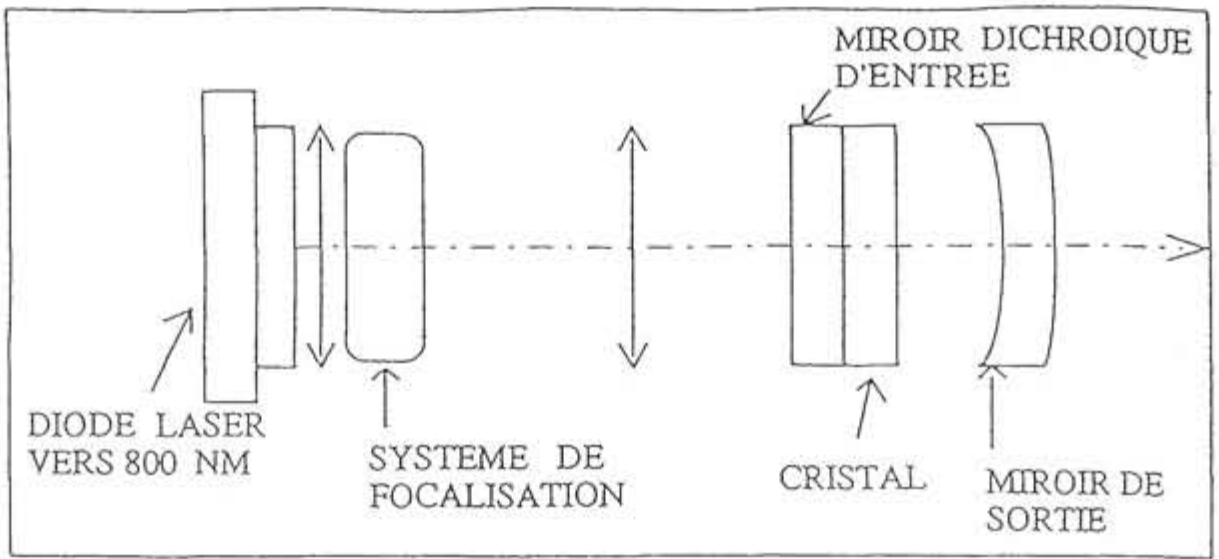

<.... $7.5 \mathrm{~mm} \mathrm{n.......}$

Figure 14c : Exemple de montage d'une cavité laser contenant un cristal $\mathrm{Y}_{3} \mathrm{Al}_{5} \mathrm{O}_{12}: \mathrm{Tm}, \mathrm{Ho}$.

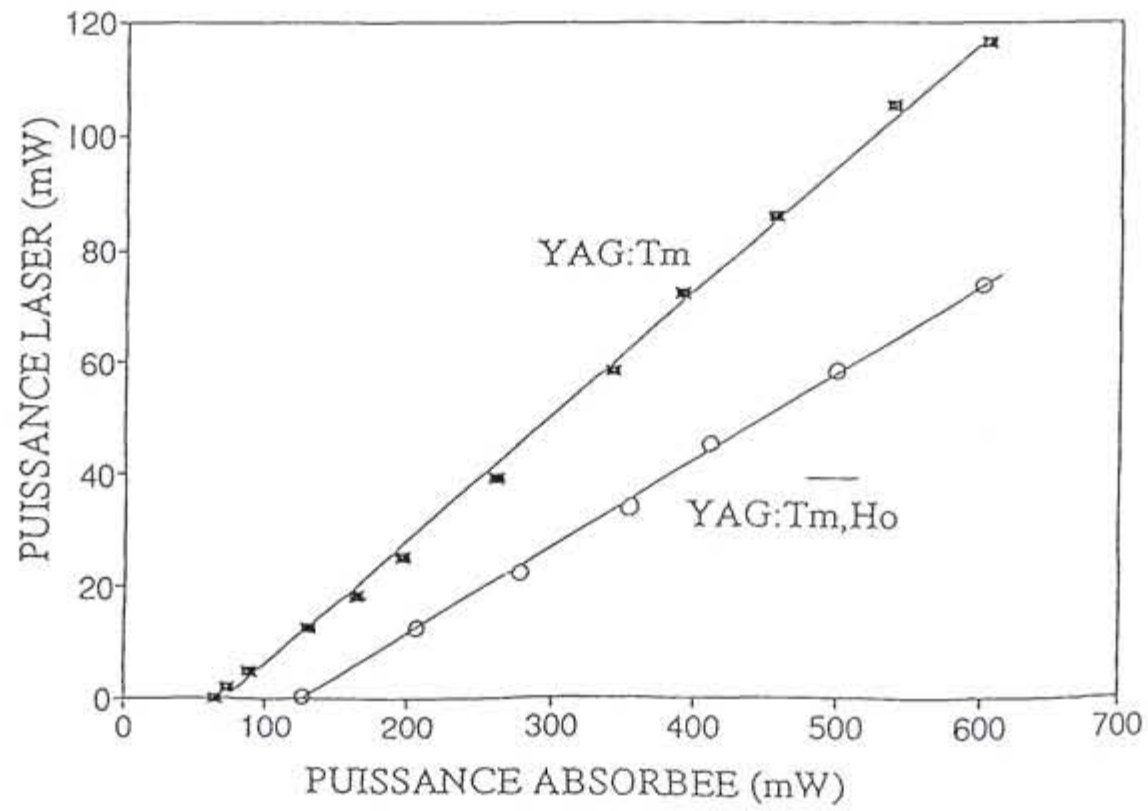

Figure 14d : Variation de la puissance laser en fonction de la puissance absorbée avec YAG:Tm et YAG:Tm, Ho. 


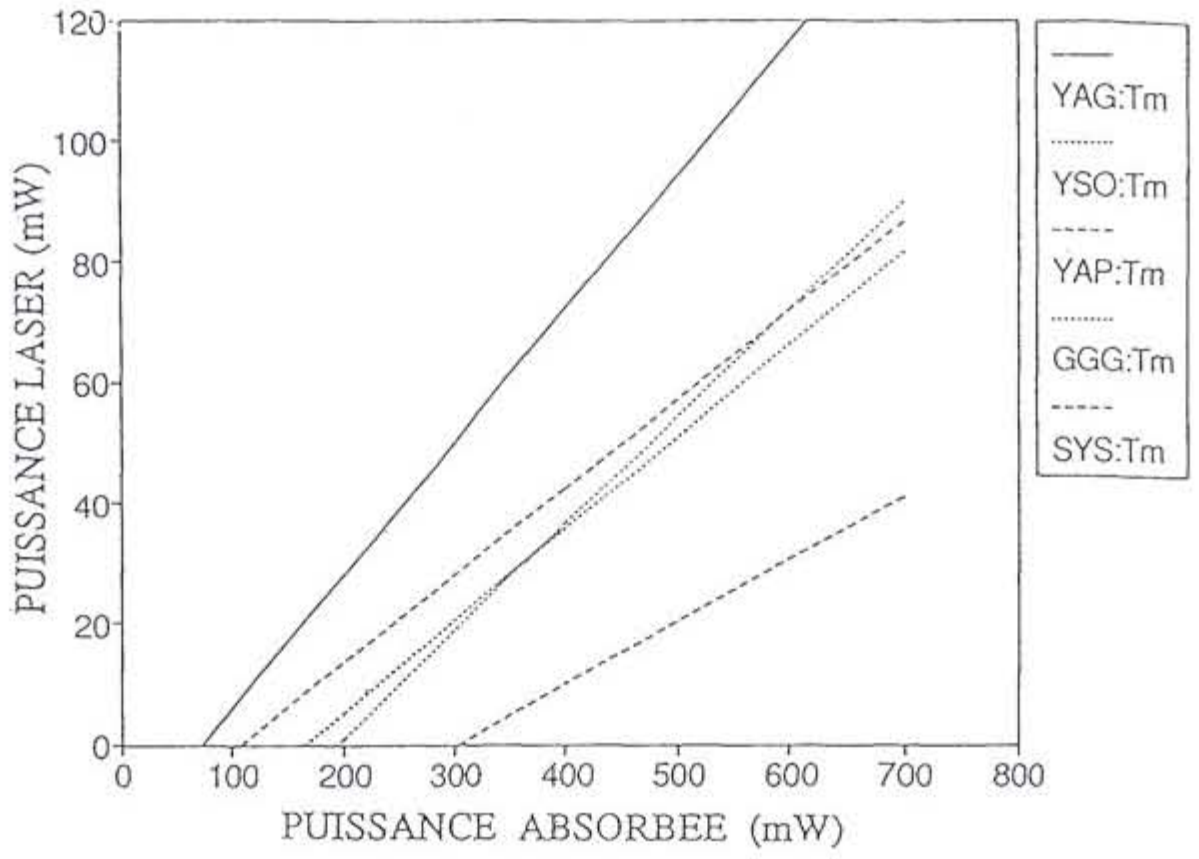

Figure 14e : Comparaison des rendements obtenus avec plusieurs cristaux dopés par l'ion $\mathrm{Tm}^{3+}$. YAG: $\mathrm{Y}_{3} \mathrm{Al}_{5} \mathrm{O}_{12}$ - YSO: $\mathrm{Y}_{2} \mathrm{SiO}_{5}$ - YAP: $\mathrm{YAlO}_{3}$ -

GGG:Gd ${ }_{3} \mathrm{Ga}_{5} \mathrm{O}_{12}-\mathrm{SYS}: \mathrm{Sr}_{2} \mathrm{Gd}_{8}\left(\mathrm{SiO}_{4}\right)_{6} \mathrm{O}_{2}$

\section{Références}

[1] Kaminskii A.A., Laser Crystals, Springer-Verlag-Berlin (1981) and (1990) Springer Series in Optical Sciences 14 and Ann. Phys. Fr 16, (1991) 639-706; Phys. Stat. Sol. (a) 148,9 (1995)

[2] Weber M.J., Lasers and Masers (Handbook of laser Science and Technology, CRC Press 1982), Vol. 1

[3] Di Bartolo B., "Spectroscopy of Solid-State Laser Type Materials " Ettore Majorana Int. Sc.Series 30 - Physical Sciences, Erice's School June 1985 (Plenum Press 1987)

[4] IEEE Journal of Quantum Electronics - Special Issue on Solid State Lasers, 24, nº (June 1988) Ed. G. Huber

[5] Proceedings of laser $\mathrm{M}_{2} \mathrm{P}$ Conference : Lyon, July 1987 (J. de Phys., 1988) ; Grenoble, July 1991 (J. de Phys IV, C7, 1991) ; Lyon, December 1993 (J. de Phys IV, C4, 1994)

[6] a) French-Israeli Worshop, "Solid State Lasers", SPIE 1182, Jerusalem, 12-14 December 1988

b) French-Israeli Worshop "Solid-State Lasers", Lyon, 5-7 December 1993, Optical Materials Ed by Reisfeld R. and Boulon G. 4, n 1, December 1994

[7] OSA Proceedings 5, "Tunable Solid State Lasers", 1-3 May 1989 in North Falmouth Cap Cod Mas ed. by Shand M.L.and Jenssen H.P.

OSA Proceedings 10, " Advanced Solid State Lasers" ed. by Dude G. and Chabe L. (1991), and 15 February 1994 in Salt Lake City, ed. by Pinto A. and Fan T. 
[8] "New Materials for advanced Solid State Lasers", Materials Research Society", Symp. Proceedings 329, ed. by Chai B., Payne S., Fan T., Cassanho A., Allik T., Boston november 29 - December 1, 1993

[9] Brinkmann U., Laser Focus World, 26 (August 1990) 27-30

[10] Moncorgé R. et al [voir 6-b]

[11] Monteil A. et al [voir 6-a]

[12] Moncorgé R. et al [voir 6-a]

[13] Payne S.A. et al, IEEE J. Quan. Electron. 24 (1989) 2243

[14] Scheps R. et al, Opt. Letters 16 (1991) 820 and Messenger H.W., Focus World 27 (November 1991) 69

[15] Moulton F., J. Opt.Soc. Amer. B3 (1986) 125

[16] Moncorgé R., Boulon G., Vivien D., Lejus A.M., Collongues R., Djévarhirdjian V., Djévarhirdjian K. and Cagnar R., IEEE J. of Quantum Electronics 24 (1988) 1049

[17] Wegner T., Hubert G. and Petertmann K. voir réf. [7], 5 (1989) 258

[18] Guyot Y., Thèse Université Lyon 1, 8 octobre 1993 n 647/93 Absorptions dans l'état excité et performances laser de cristaux dopés par l'ion néodyme.

[19] Marqueville G. et al, Opto 62, 35 (1991)

[20] Kalisky Y., voir [6-a]

[21] Vivien D., voir [6-a]

[22] Springer J., Clausen R., Huber G., Peterman K.et Mateika D., (voir ref 7bis p. 346)

[23] Moncorgé R. et al, J. de Physique IV, C4-377 (1994).

[24] Kaminskii A., Butashin A., Mill B., Boulon G., Moncorgé R. and Garcia-Solé J. Phys. Stat. Sol.(a) 139 K 133 (1993).

[25] Moncorgé R., Manaa H., Boulon G - voir [6-b] p 139 - 151 